\title{
A multiclade env-gag VLP mRNA vaccine elicits tier-2 HIV-1-neutralizing antibodies and reduces the risk of heterologous SHIV infection in macaques
}

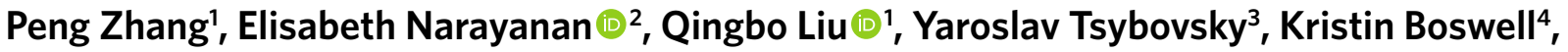 \\ Shilei Ding ${ }^{5}$, Zonghui Hu, Dean Follmann6, Yin Lin1, Huiyi Miao', Hana Schmeisser', Denise Rogers', \\ Samantha Falcone², Sayda M. Elbashir², Vladimir Presnyak², Kapil Bahl², Madhu Prabhakaran", \\ Xuejun Chen ${ }^{4}{ }^{4}$, Edward K. Sarfo ${ }^{4}{ }^{4}$, David R. Ambrozak ${ }^{4}$, Rajeev Gautam7, Malcom A. Martin ${ }^{7}$, \\ Joanna Swerczek ${ }^{8}$, Richard Herbert ${ }^{8}$, Deborah Weiss ${ }^{9}$, Johnathan Misamore ${ }^{9}$, Giuseppe Ciaramella², \\ Sunny Himansu ${ }^{1}{ }^{2}$, Guillaume Stewart-Jones ${ }^{2}$, Adrian McDermott ${ }^{1}{ }^{4}$, Richard A. Koup ${ }^{4}$, \\ John R. Mascola ${ }^{4}$, Andrés Finzi ${ }^{5}$, Andrea Carfi ${ }^{2}$, Anthony S. Fauci ${ }^{1}$ and Paolo Lusso ${ }^{1 凶}$
}

The development of a protective vaccine remains a top priority for the control of the HIV/AIDS pandemic. Here, we show that a messenger RNA (mRNA) vaccine co-expressing membrane-anchored HIV-1 envelope (Env) and simian immunodeficiency virus (SIV) Gag proteins to generate virus-like particles (VLPs) induces antibodies capable of broad neutralization and reduces the risk of infection in rhesus macaques. In mice, immunization with co-formulated env and gag mRNAs was superior to env mRNA alone in inducing neutralizing antibodies. Macaques were primed with a transmitted-founder clade-B env mRNA lacking the N276 glycan, followed by multiple booster immunizations with glycan-repaired autologous and subsequently bivalent heterologous envs (clades $A$ and $C$ ). This regimen was highly immunogenic and elicited neutralizing antibodies against the most prevalent (tier-2) HIV-1 strains accompanied by robust anti-Env CD4 ${ }^{+} \mathrm{T}$ cell responses. Vaccinated animals had a $79 \%$ per-exposure risk reduction upon repeated low-dose mucosal challenges with heterologous tier-2 simian-human immunodeficiency virus (SHIV AD8). Thus, the multiclade env-gag VLP mRNA platform represents a promising approach for the development of an HIV-1 vaccine.

$\Lambda$ lthough more than four decades have elapsed since the onset of the HIV/AIDS pandemic, a protective vaccine has yet to be developed. A series of major hurdles are responsible for this lack of success, including the remarkable antigenic variability of the HIV-1 envelope (Env), the protected configuration of conserved neutralization epitopes due to both an extensive glycan shield and conformational masking, the relative in vivo rarity of B cells expressing germline precursors of broadly neutralizing antibodies (bNAbs), and the autoreactivity of bNAbs directed against certain Env sites ${ }^{1-3}$. In the context of this unique combination of challenges, elicitation of bNAbs has proven extremely difficult to achieve by vaccination, and even natural HIV-1 infection leads to broad neutralization only in a fraction of infected individuals and only after months or years of persistent antigenic stimulation ${ }^{1,3}$. Accordingly, most bNAbs that have been isolated from chronically infected individuals have unusual features, such as a high frequency of somatic hypermutations and long complementarity-determining regions $\mathrm{s}^{4,5}$.

A variety of HIV-1 vaccine approaches have been evaluated in preclinical models and clinical studies, but the results have been, overall, unsatisfactory ${ }^{6-8}$. Only a single human vaccine trial, the RV144 study in Thailand, has hitherto yielded a low degree of protec- tion ( 30\%), although it failed to elicit bNAbs and its partial success was not reproduced in recent attempts at corroboration in clinical trials conducted in Africa ${ }^{9-11}$. Preclinical studies with soluble forms of the HIV-1 Env trimer stabilized in a configuration similar to that presented on native viral particles, such as SOSIP trimers and variations thereof ${ }^{12-17}$, have documented robust induction of autologous neutralizing antibodies, primarily targeting strain-specific holes in the Env glycan shield, accompanied by large amounts of off-target, non-neutralizing antibodies primarily directed at the trimer base, further emphasizing the challenges in eliciting bNAbs by vaccina$\operatorname{tion}^{18-23}$. Epitope-based vaccine approaches, guided by knowledge of the major structural vulnerability supersites recognized by bNAbs, have been extensively explored ${ }^{24,25}$ and recently yielded a partial success in inducing neutralization breadth by targeting the fusion peptide in glycoprotein (gp)41 (refs ${ }^{26,27}$ ). Another major line of research, based on sequential modulation of B cell responses along specific bNAb lineages by initial priming with germline-engaging core antigens followed by stepwise immunization with increasingly antibody-shielded Env forms, holds promise of success in recruiting the appropriate precursor $\mathrm{B}$ cells $^{28-30}$. Additional vaccine strategies, mostly based on prime-boost schemes, have yielded different

'Laboratory of Immunoregulation, NIAID, NIH, Bethesda, MD, USA. ${ }^{2}$ Moderna Inc., Cambridge, MA, USA. ${ }^{3}$ Cancer Research Technology Program, Leidos Biomedical Research, Inc., Frederick National Laboratory for Cancer Research, Frederick, MD, USA. ${ }^{4}$ Vaccine Research Center, NIAID, NIH, Bethesda, MD, USA. ${ }^{5}$ Université de Montreal, Montreal, Quebec, Canada. ${ }^{6}$ Biostatistics Research Branch, NIAID, NIH, Bethesda, MD, USA. ${ }^{7}$ Laboratory of Molecular Microbiology, NIAID, NIH, Bethesda, MD, USA. ${ }^{8}$ Experimental Primate Virology Section, NIAID, Poolesville, MD, USA. ${ }^{9}$ Bioqual Inc., Rockville, MD, USA. 凶e-mail: plusso@niaid.nih.gov 
degrees of protection in non-human primate models ${ }^{31-35}$. Some approaches have used messenger RNA (mRNA) as a vector, resulting in the induction of polyfunctional antibody responses comparable to those induced by protein immunization, as well as efficient T cell responses ${ }^{36-38}$. Altogether, the evidence so far accumulated suggests that no individual factor will determine the ultimate success of a bNAb-inducing HIV-1 vaccine, which probably requires a combination of efficient precursor B cell priming, optimization of Env design and presentation, and sustained heterologous Env boosting.

\section{Results}

Design of an env-gag VLP mRNA vaccine platform. Critical advancements in mRNA technology over the past two decades ${ }^{39,40}$ have enabled the development of mRNA-based vaccine platforms, which have recently shown remarkable effectiveness against COVID-19 (refs ${ }^{41-43}$ ). Taking advantage of the versatility of mRNA as an expression system, we designed a novel vaccine platform by combining a series of features that we postulated to be critical for the elicitation of protective antibody responses. These include, first, the use of mRNA as a vehicle in order to instruct host cells to endogenously express membrane-bound viral glycoproteins and decorate them with native $N$-linked glycosylation; second, the use of full-length or minimally truncated HIV-1 Envs that do not expose distractive immunodominant epitopes, unlike truncated soluble trimers; third, co-expression of Env with Gag in order to promote the in vivo production of virus-like particles (VLPs), which closely mimic native viral particles produced by HIV-1 infection; fourth, initial priming with an Env capable of engaging germline bNAb precursors; and last, intensive heterologous boosting with tier-2 Envs from different clades in order to selectively expand $\mathrm{B}$ cell responses against shared bNAb epitopes and mimic the sustained antigenic stimulation that occurs in chronically infected patients who eventually develop broad neutralization ${ }^{1-5}$.

Co-expression of env and gag mRNAs leads to VLP production. The production of VLPs may offer critical advantages in terms of both antigen structure and size, and was suggested to be best suited for efficient uptake and processing by antigen-presenting cells ${ }^{44-47}$. Previous work showed that expression of lentiviral Gag proteins is sufficient to induce the extracellular release of Gag-containing VLPs $^{48,49}$, which can mount viral Env glycoproteins on their surface when co-expressed in the same cell ${ }^{50}$. We evaluated the ability of different HIV-1 Env immunogens to generate extracellular VLPs by in vitro mRNA transfection into female human embryonic kidney (HEK)293T cells. Thus, mRNA expressing Env from five HIV-1 strains of different clades (BG505 375Y, clade A; WITO.27 $\Delta 276$ and WITO.27 G153E, clade B; 426c.D276.D460.D463 (426c.3dg) and DU422 375Y, clade C) were individually co-transfected with simian immunodeficiency virus (SIV) gag mRNA (strain SIVmac239). SIV, rather than HIV-1, Gag was used given that the preclinical studies involved macaques, which are susceptible to infection with chimeric simian-human immunodeficiency virus (SHIV) but not HIV1. After 48 hours, the production of VLPs was tested in the culture supernatants using a virion-capture assay with two bNAbs (PG16 and PGT128) bound to immunomagnetic beads, and the amount of captured SIV p27 $7_{\text {Gag }}$ was quantified. The assembly and extracellular release of Env-coated VLPs were readily documented with all the Envs tested, while only background levels were measured when Gag was expressed alone (Fig. 1a). These results confirmed that co-expression of env and gag mRNAs leads to the production and extracellular release of Env-coated VLPs.

Characteristics of $m R N A$-expressed Env-Gag VLPs. To characterize the structure of mRNA-expressed Gag-Env VLPs, we performed negative-staining electron microscopy (NSEM) on concentrated

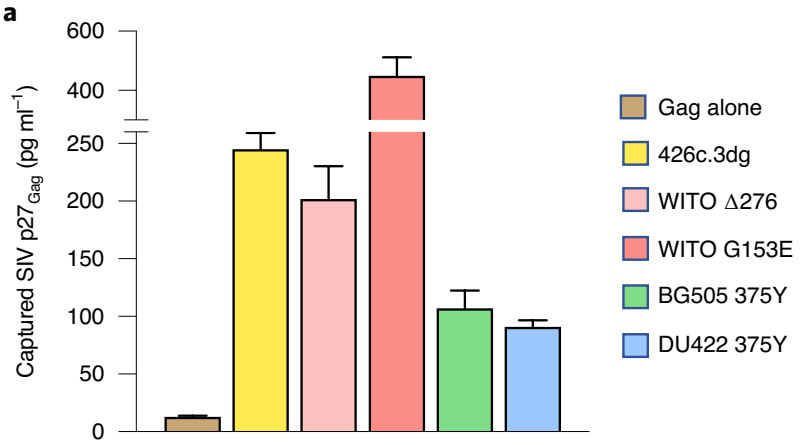

b
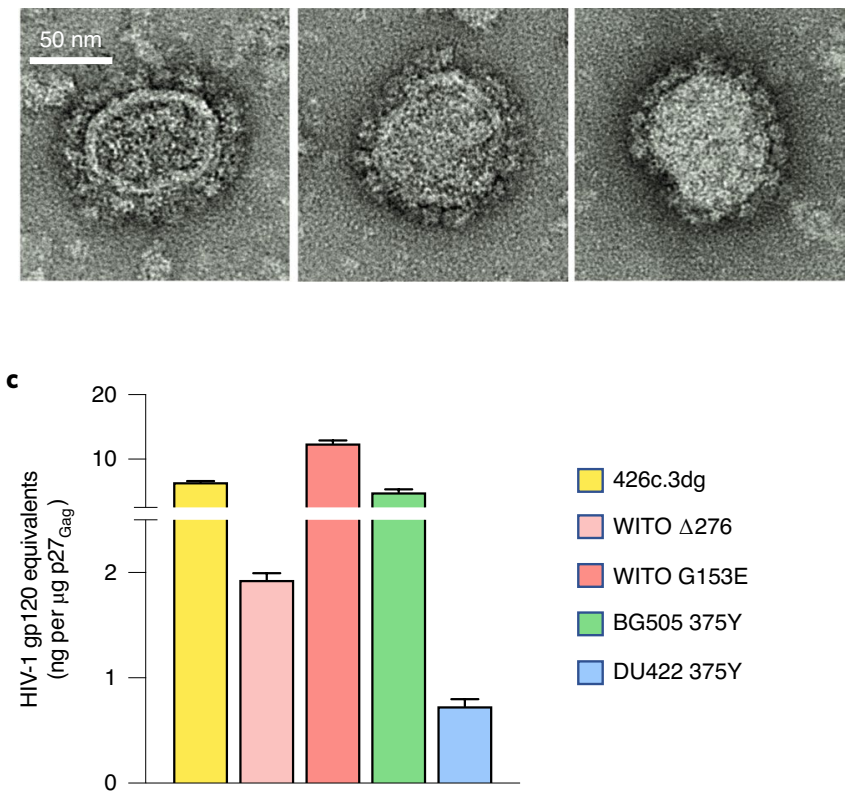

Fig. 1 | Production and characteristics of mRNA-expressed Env-Gag VLPs. a, Co-transfection of different HIV-1 env mRNA with SIV gag mRNA leads to the assembly and extracellular release of VLPs. Quantification of released VLPs in the supernatants of transfected cells was done by virion-capture assay using magnetic beads coated with two anti-Env human bNAbs (PG16 and PGT128) and then by measuring the amount of captured SIV Gag by ELISA. Data given as the mean \pm s.d. of three replicates from a representative experiment out of three performed. b, Visualization of three representative mRNA-expressed Env-Gag VLPs using NSEM. The conservatively inferred mean number of spikes per particle is $32.4 \pm 10.1$. c, Quantification of HIV-1 Env in mRNA-expressed VLPs. This was done using a trimer-specific ELISA with a cocktail of anti-Env human bNAbs (that is, PG16, PGT128, N6, PGT151). To obtain a reliable quantification, a reference curve was generated for each HIV-1 Env using the corresponding soluble trimers. The amount of HIV-1 Env was normalized for the amount of SIV Gag, as quantified by ELISA. Data given as the mean \pm s.d. of three replicates from a representative experiment out of three performed.

VLPs after ultracentrifugation on a sucrose cushion. The VLPs appeared as roughly spherical profiles with a mean diameter of $\sim 90 \mathrm{~nm}$ (range, 65-110 $\mathrm{nm}$ ), an external membrane surrounding an amorphous matrix and a dense array of spike-like projections on the surface (Fig. 1b). The projections visible around the profile of VLPs were manually counted and their number was doubled to conservatively account for invisible spikes on the top and bottom surfaces of the VLPs. Using this method, the inferred mean number of spikes per particle was $32.4 \pm 10.1$, which is probably an 
underestimate, but still more than twice the number of spikes present on wild-type HIV-1 virions ${ }^{51}$.

To evaluate the relative abundance of Env in VLPs produced with different HIV-1 Env immunogens, we performed a quantitative analysis of concentrated VLP stocks by ELISA, using a cocktail of four bNAbs reactive with all the Envs used (that is, PG16, PGT128, N6, PGT151). Env incorporation was detected in all VLPs, with variable amounts of gp120 equivalents relative to SIV Gag p27, ranging from 0.7 to $12.5 \mathrm{ng}$ per $\mu \mathrm{g} \mathrm{p} 27_{\text {Gag }}$ (Fig. 1c).

Comparison of $e n v$-only versus $e n v$ - $g a g$ mRNA vaccination in mice. Based on the in vitro evidence of VLP assembly and extracellular release upon Env-Gag co-expression, we compared the in vivo immunogenicity of HIV-1 env plus SIV gag mRNA versus HIV-1 env mRNA alone in a wild-type mouse model. The study design, which included three groups of mice, is summarized in Fig. 2a: group $1(n=8)$ was inoculated with HIV-1 env mRNA (strain $426 \mathrm{c} .3 \mathrm{dg})$; group $2(n=8)$, with the same env mRNA co-formulated with SIV gag mRNA (strain SIVmac239); and group $3(n=4)$, with PBS as a control. To ensure co-expression of Env and Gag in the same cells, env and gag mRNAs were co-formulated in the same lipid nanoparticles (Fig. 2b). The antigenic profile of the mRNA-expressed 426c.3dg Env is given in Supplementary Table 1. All the mice received two inoculations 28 days apart, and blood was drawn at 2 week intervals until day 57 . The vaccine was immunogenic, as shown by the appearance of trimer-binding antibodies after a single immunization (days 14 and 28), which were markedly boosted at weeks 2 (day 43) and 4 (day 57) after the second immunization (Fig. 2c). Despite a higher mean titer in group 2 than in group 1 at both day 14 and day 28, the difference did not reach statistical significance. Neutralizing antibodies against the autologous virus, $426 \mathrm{c} .3 \mathrm{dg}$, appeared earlier and were boosted to higher levels in group 2 compared with group 1 (Fig. $2 \mathrm{~d}$ and Supplementary Table 2 ). The mean ( \pm s.e.m.) reciprocal half-maximal inhibitory concentration $\left(\mathrm{IC}_{50}\right)$ at day 57 was $10,955 \pm 3,949$ for $e n v-g a g$ mRNA and $1,920 \pm 2,476$ for env-only mRNA $(P=0.042$, two-tailed unpaired $t$-test). Notably, all the mice $(8 / 8,100 \%)$ immunized with env-gag mRNA developed measurable neutralizing antibodies by day 57 , in contrast with only five of eight mice (62.5\%) immunized with env mRNA alone and none of the controls (Fig. 2e). These data indicate that a vaccine based on co-formulated env-gag mRNA is superior to $e n v$-only mRNA in inducing neutralizing antibody responses, and provide an in vivo validation of the env-gag mRNA VLP vaccine platform.

An env-gag VLP mRNA vaccine is safe and immunogenic in macaques. We next assessed the immunogenicity and efficacy of a VLP-producing env-gag mRNA vaccine in rhesus macaques, which provide a more suitable preclinical model and can be challenged with live SHIV bearing the HIV-1 Env. The design of the study is summarized in Fig. 3a. A total of 14 naive female rhesus macaques ( 7 vaccinees and 7 controls) were enrolled, all negative for the protective Mamu alleles $A^{\star} 01, B^{\star} 08$ and $B^{\star} 17$. Vaccinated animals sequentially received four env mRNAs (that is, WITO4160.27 $\Delta 276$, WITO4160.27G153E, BG505.T332N and DU422.1) from three different HIV-1 clades, each co-formulated with SIVmac239 gag mRNA at a ratio between 1.7:1 and 2.2:1. In previous in vitro transfection experiments we had determined that a ratio of 2:1 was optimal for VLP formation. A variable amount of total mRNA was inoculated at each immunization (up to $400 \mu \mathrm{g}$ per dose) depending on the production yield (Supplementary Table 3 ). The antigenic profile of the four Env immunogens is given in Supplementary Table 1. All the Envs, except BG505.T332N, were truncated at residue 745 in the cytoplasmic tail of gp41 to increase the level of cell-surface expression and, thereby, VLP incorporation without altering their antigenic profile. The WITO4160 clone $27 \mathrm{Env}$ (WITO.27, clade
B) $)^{52}$ was selected because it is a transmitted-founder Env that efficiently presents all major bNAb epitopes, binds to the unmutated common ancestor of the bNAb CH01 (ref. ${ }^{53}$ ), and naturally binds to and utilizes macaque CD4 (Supplementary Table 1 and Extended Data Fig. 1). The initial priming was performed with an open form of WITO.27 $(\Delta 276)$ from which the $N$-glycan at position 276 in gp120 was removed to facilitate recognition of the CD4-binding site (CD4-BS) by putative germline antibody precursors ${ }^{54}$. The two subsequent boosts were performed with a closed form of WITO.27 bearing the $\mathrm{N} 276$ glycan and a $\mathrm{G} \rightarrow \mathrm{E}$ substitution at position 153 , which was introduced because it is present in most reported WITO4160 clones and reduces the exposure of CD4-induced epitopes (Supplementary Table 4). The WITO SOSIP.664 trimer used as a protein boost in vaccine subgroup 1 had the same N276.G153E sequence. The BG505.W6M.C2.T332N.375Y Env (BG505, clade A) was selected because it is a reference Env in the field, is available in a SOSIP trimer version for protein boosting, and binds to the majority of known bNAbs but inefficiently to non-neutralizing antibodies (Supplementary Table 1). The DU422.1.386N.295N.375Y Env (DU422, clade C) was selected because it is available in a SOSIP trimer version and has a closed trimer conformation with minimal exposure of epitopes for non-neutralizing antibodies (Supplementary Table 1). Both the BG505 and DU422 Envs and their respective SOSIP versions were modified by an $\mathrm{S} \rightarrow \mathrm{Y}$ substitution at position 375 to allow binding to macaque CD4 (ref. ${ }^{55}$ ), which is otherwise null (Extended Data Fig. 1). This choice was made to better mimic the physiological conditions that occur in humans, given that the use of HIV-1 Envs in macaques could yield artifactually more favorable results by averting in vivo CD4 interaction and CD4-induced conformational changes. The S375Y mutation did not significantly alter the antigenic profile of these two Envs (Supplementary Table 1$)^{55}$.

The original vaccine protocol encompassed seven sequential immunizations: three with WITO.27 Env, defined as the autologous phase, and four with combined BG505 and DU422 Envs, defined as the mixed heterologous phase. To exclude the possibility of generating hybrid trimers containing the two Envs, the two heterologous immunogens were inoculated at two distinct sites at least 1 inch apart in the same muscle. Given that mRNA vaccines have only recently been introduced, we wanted to address the importance of protein boosts in addition to mRNA. Thus, one subgroup of animals (subgroup $1 ; n=3$ ) received a single protein boost with homologous SOSIP trimers at the end of each phase, while the other (subgroup 2; $n=4$ ) received mRNA exclusively during both phases. After completion of the seven immunizations, however, a protocol amendment was introduced to perform three additional boosts in an attempt to further enhance the neutralizing antibody titers before the live virus challenge: one, at week 56, with the autologous Env (WITO.27; either SOSIP trimer or mRNA in subgroups 1 and 2 , respectively) and two with recombinant heterologous SOSIP trimers in all seven animals (one with JR-FL SOSIP.664 at week 51 and one with a mixture of four trimers, that is, WITO, BG505, DU422 and JR-FL SOSIP.664, at week 59). Thus, altogether, subgroup 1 received five mRNA and five protein inoculations, while subgroup 2 received eight mRNA and only two protein inoculations.

Despite the multiple immunizations with high mRNA doses, the vaccine was well tolerated with only mild adverse events after each inoculation (Supplementary Table 5). The most common side-effect was loss of appetite, while local reactions such as swelling or redness at the injection site were notably absent. The env-gag VLP mRNA vaccine was highly immunogenic, as shown by the rapid induction of autologous trimer-binding antibodies in all macaques as early as week 6 , following a single mRNA inoculation (Fig. $3 \mathrm{~b}$ and Supplementary Table 6). The antibody titers markedly increased after boosting with the closed WITO Env at weeks 11 and 19. Given that the induction of antibodies against the V3 loop has been highlighted 
a

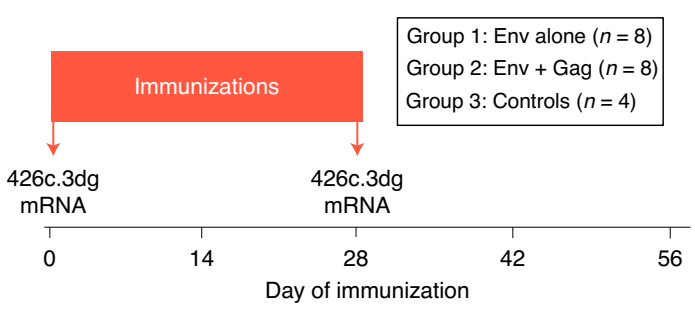

b

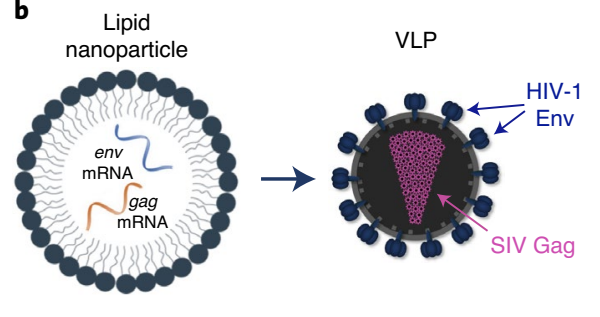

c

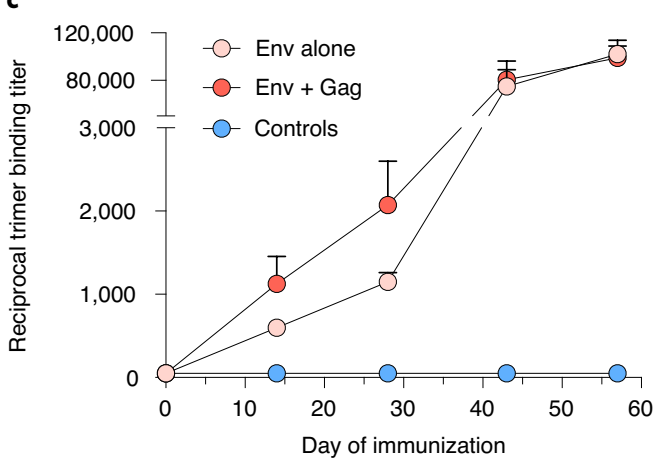

d

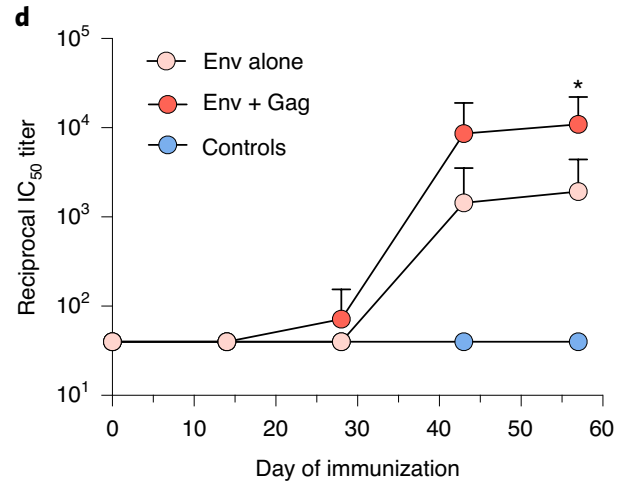

e
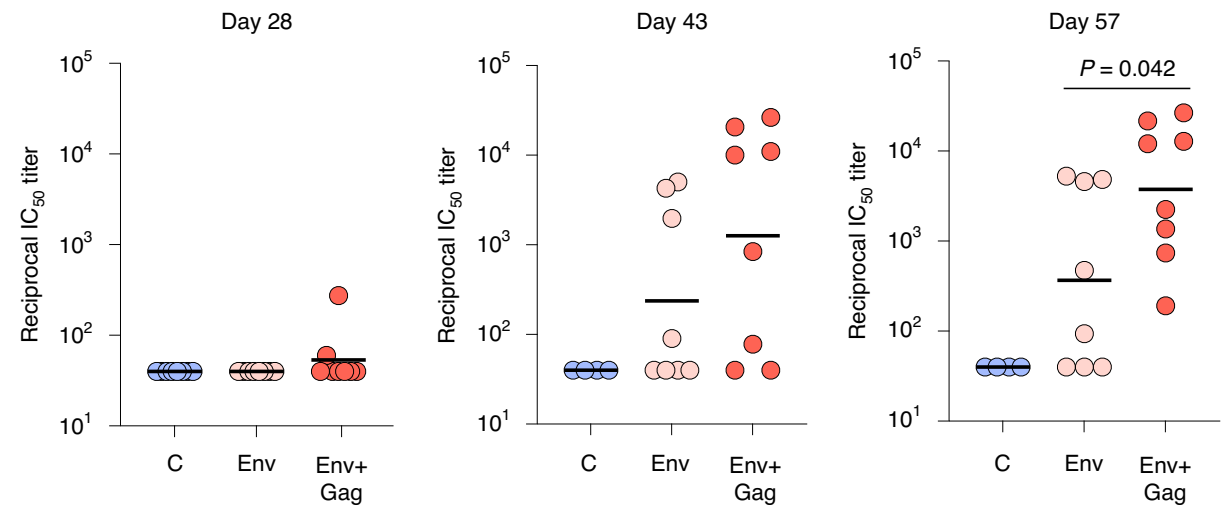

Fig. 2 | Immunogenicity and efficacy of env-only versus env-gag mRNA vaccines in mice. a, Timeline of immunization. Balb-c mice ( $n=8$ per group) were immunized at two time points, week 0 and week 4, with mRNA either expressing the HIV-1 426c.3dg Env alone (group 1) or co-expressing HIV-1 426c.3dg Env and SIVmac239 Gag (group 2); a third group ( $n=4)$ was inoculated with PBS as a control (group 3). HIV-1 Env 426c.3dg is a germline antibody-engager clade-C Env that lacks three critical glycans (N276, N460, N463) that protect the CD4-BS. b, Schematic representation of the env-gag mRNA immunogen structure. Co-formulation of HIV-1 env mRNA with SIV gag mRNA leads to the assembly and release of VLPs. $\mathbf{c}$, Time-course of HIV-1 Env trimer binding by sera from mice immunized with mRNA expressing HIV-1 Env only or co-expressing HIV-1 Env and SIV Gag, or controls inoculated with PBS. Mean reciprocal endpoint dilution titers ( \pm s.e.m.) from each study group (group 1 and $2, n=8$; group $3, n=4$ ) are shown at day 14 and 28 (2 and 4 weeks, respectively, after the first immunization), and at day 43 and 57 ( 2 and 4 weeks, respectively, after the second immunization). Although at days 14 and 28 the mean titers were higher in group 2 than in group 1, the difference did not reach statistical significance (two-tailed unpaired $t$-test). Each serum was tested in duplicate wells. d, Time-course of HIV-1426c-3dg neutralization by sera from mice immunized with mRNA expressing HIV-1

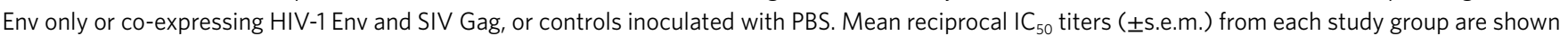
at day 0 (pre-immune), 14, 28, 43 and 57. ${ }^{\star} P<0.05$ between groups 1 and 2 . Each serum was tested in duplicate wells. Infection controls in the absence of serum were tested in six replicate wells. e, Neutralization of HIV-1 426c.3dg by sera from individual mice immunized with mRNA expressing HIV-1 Env only or co-expressing HIV-1 Env and SIV Gag, or controls inoculated with PBS, at day 28, 43 and 57 . The geometric mean for each group is indicated by the horizontal black lines. The difference between group 1 and group 2 at day 57 was statistically significant (two-tailed unpaired $t$-test). Each serum was tested in duplicate wells. Infection controls in the absence of serum were tested in six replicate wells. The mouse immunization study was performed once.

as a marker of non-protective vaccine responses ${ }^{18}$, we tested the reactivity with a linear $\mathrm{V} 3$ peptide containing the autologous sequence (WITO pV3). Anti-V3 antibody titers were generally lower and developed with slower kinetics compared with trimer-binding antibodies (Fig. 3c), suggesting that the bulk of trimer-binding antibodies elicited by the vaccine were not directed against the V3 loop. Heterologous trimer-binding antibodies, tested against the AD8 trimer, were induced in all animals, although they emerged later and remained at lower titers compared with autologous trimer-binding antibodies, reaching the highest levels only after completion of the four heterologous immunizations (Fig. 3d). No significant differences in the magnitude and kinetics of antibody responses against autologous or heterologous trimers or the V3 loop were observed between animals in subgroups 1 and 2 (Extended Data Fig. 2). 
a

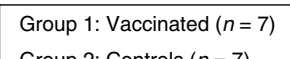

Group 2: Controls $(n=7)$

\begin{tabular}{|c|c|c|c|c|c|c|c|c|c|}
\hline \multicolumn{3}{|c|}{$\begin{array}{l}\text { Autologous Env } \\
\text { (clade B) }\end{array}$} & \multicolumn{4}{|c|}{$\begin{array}{l}\text { Mixed heterologous } \\
\text { Envs (clades } A+C \text { ) }\end{array}$} & $\frac{T}{\Phi}$ & 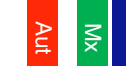 & $\begin{array}{l}\text { Mucosal SHIV } \\
\text { AD8 challenges }\end{array}$ \\
\hline$\downarrow$ & $\downarrow$ & $\downarrow$ & $\downarrow$ & $\downarrow$ & $\downarrow$ & $\downarrow$ & $\downarrow$ & $\downarrow$ & $\downarrow \downarrow \downarrow \downarrow \downarrow \downarrow \downarrow \downarrow \downarrow \downarrow \downarrow$ \\
\hline $\begin{array}{c}\text { WITO } \\
\Delta 276 \\
\text { mRNA }\end{array}$ & $\begin{array}{l}\text { WITO } \\
\text { mRNA }\end{array}$ & $\begin{array}{l}\text { WITO } \\
\text { mRNA } \\
\text { or } \\
\text { protein }\end{array}$ & $\begin{array}{c}\text { BG505 } \\
\text { +DU422 } \\
\text { mRNA }\end{array}$ & $\begin{array}{c}\text { BG505 } \\
+ \text { DU422 } \\
\text { mRNA }\end{array}$ & $\begin{array}{c}\text { BG505 } \\
\text { +DU422 } \\
\text { mRNA }\end{array}$ & $\begin{array}{c}\text { BG505 } \\
\text { +DU422 } \\
\text { mRNA } \\
\text { or } \\
\text { protein }\end{array}$ & 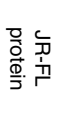 & 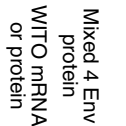 & \\
\hline 0 & 11 & 19 & 27 & 35 & 43 & 47 & 51 & 56 & 72 \\
\hline
\end{tabular}
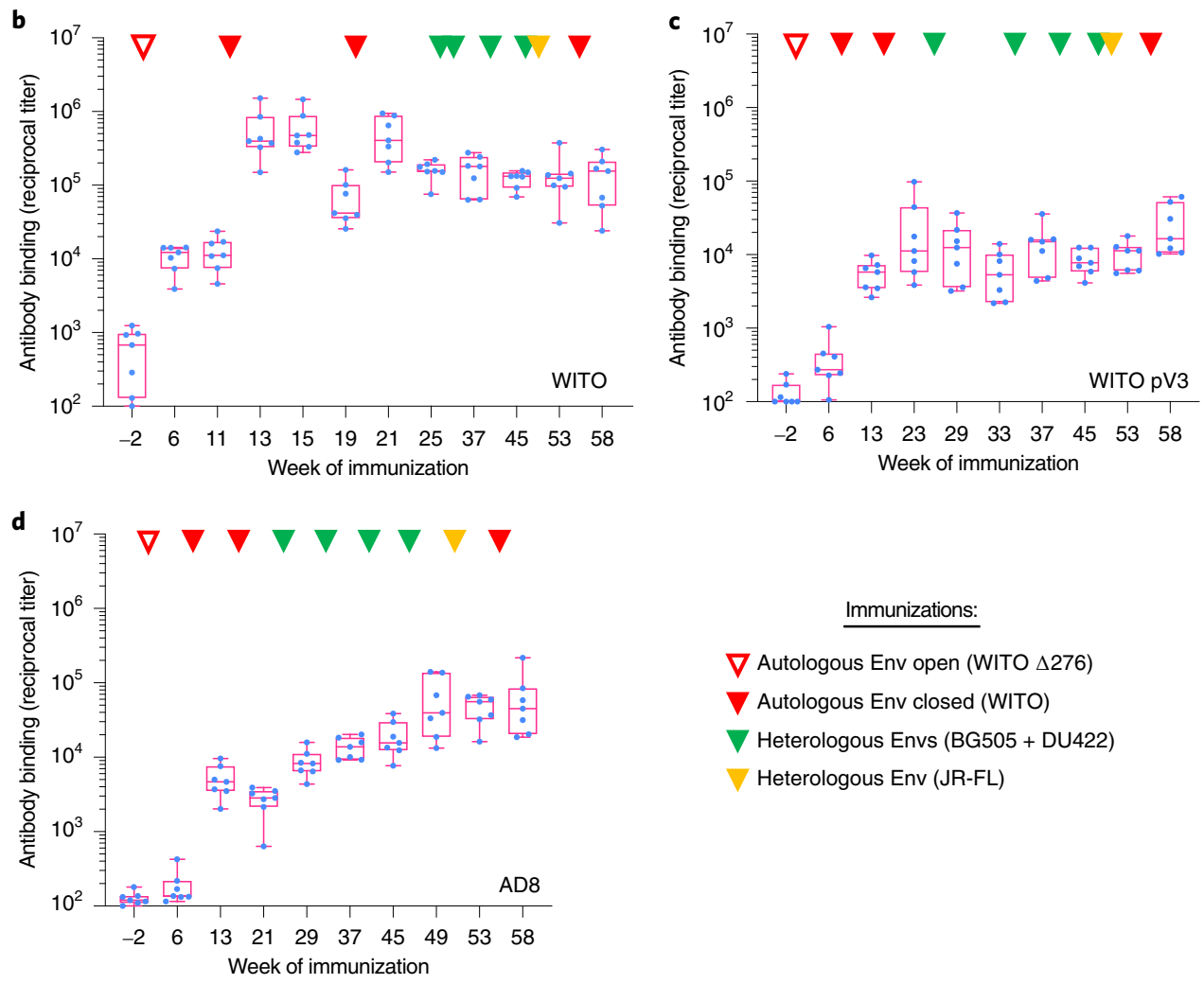

Fig. 3 | Immunogenicity of an env-gag VLP mRNA vaccine in macaques. a, Timeline of immunization and live virus challenge. A total of 14 naive female rhesus macaques ( 7 vaccinees and 7 naive controls) were enrolled, all negative for the protective Mamu alleles $A^{\star} 01, B^{\star} 08$ and $B^{\star} 17$. Vaccinated animals received four env mRNAs from three different HIV-1 clades (that is, WITO4160 $\Delta 276$, WITO4160, BG505.T332N and DU422.1), each co-formulated with SIVmac239 gag mRNA at env: gag ratios ranging between 1.7:1 and 2.2:1. The original study design included seven sequential immunizations (three with the WITO.27 Env, defined as the autologous phase, and four with combined BG505 and DU422 Envs, defined as the mixed heterologous phase). Although all the animals received mRNA, they were further subdivided into two subgroups based on the final boost in each of these phases: one subgroup received seven mRNA immunizations while the other received mRNA followed by a single protein boost with homologous SOSIP trimers in each phase. After completion of the first seven immunizations, a protocol amendment was introduced to include three additional immunizations: one with the autologous Env (WITO.27 mRNA or protein in the respective subgroups) and two with recombinant heterologous proteins in both subgroups (one at week 51 with JR-FL SOSIP.664 and one at week 59 with a mixture of four soluble trimers, that is, WITO SOSIP.664, BG505 SOSIP.664, DU422 SOSIP.664 and JR-FL SOSIP.664). b, Induction of autologous trimer-binding antibodies in all seven immunized macaques over time as assayed with ELISA using the WITO SOSIP.664 trimer captured on lectin-coated plates. The data are given in box-and-whisker representation, with the ends of the box representing the first and third quartiles, the horizontal line representing the median, and the whiskers representing the minimum and maximum, with the blue dots representing endpoint dilution titers for individual macaques. Each serum was tested in duplicate wells. c, Induction of antibodies binding to the autologous V3 loop in immunized macaques over time as assayed using a linear WITO V3 peptide directly coated on the plate surface. Each serum was tested in duplicate wells. d, Induction of antibodies binding to a heterologous trimer (AD8) in immunized macaques over time as assayed with ELISA using the soluble AD8 trimer captured on lectin-coated plates. The sequence and timing of immunizations are indicated by color-coded triangles on the top. Each serum was tested in duplicate wells.

Neutralizing antibody responses. Next, we evaluated the elicitation of neutralizing antibodies. Low titers of autologous neutralizing antibodies (against wild-type WITO.27 bearing the N276 glycan) became detectable as early as 2 weeks after the second autologous immunization (week 13), but they were initially short-lived and disappeared in all animals by week 19 (Fig. 4a and Supplementary Table 7). 
a

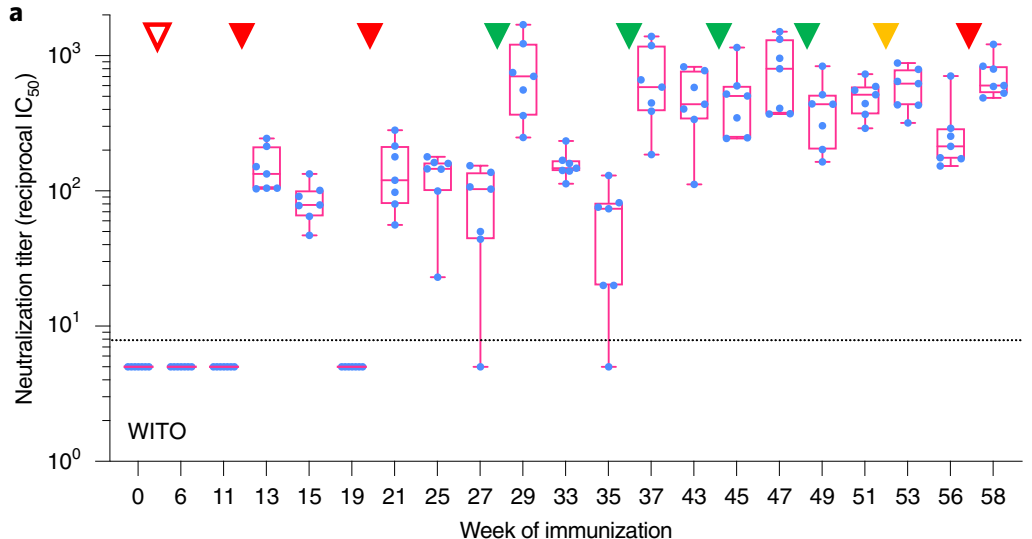

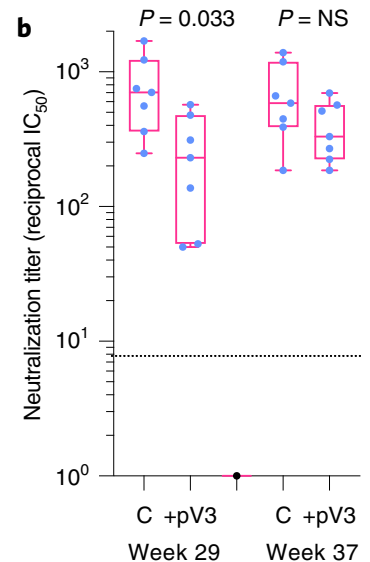
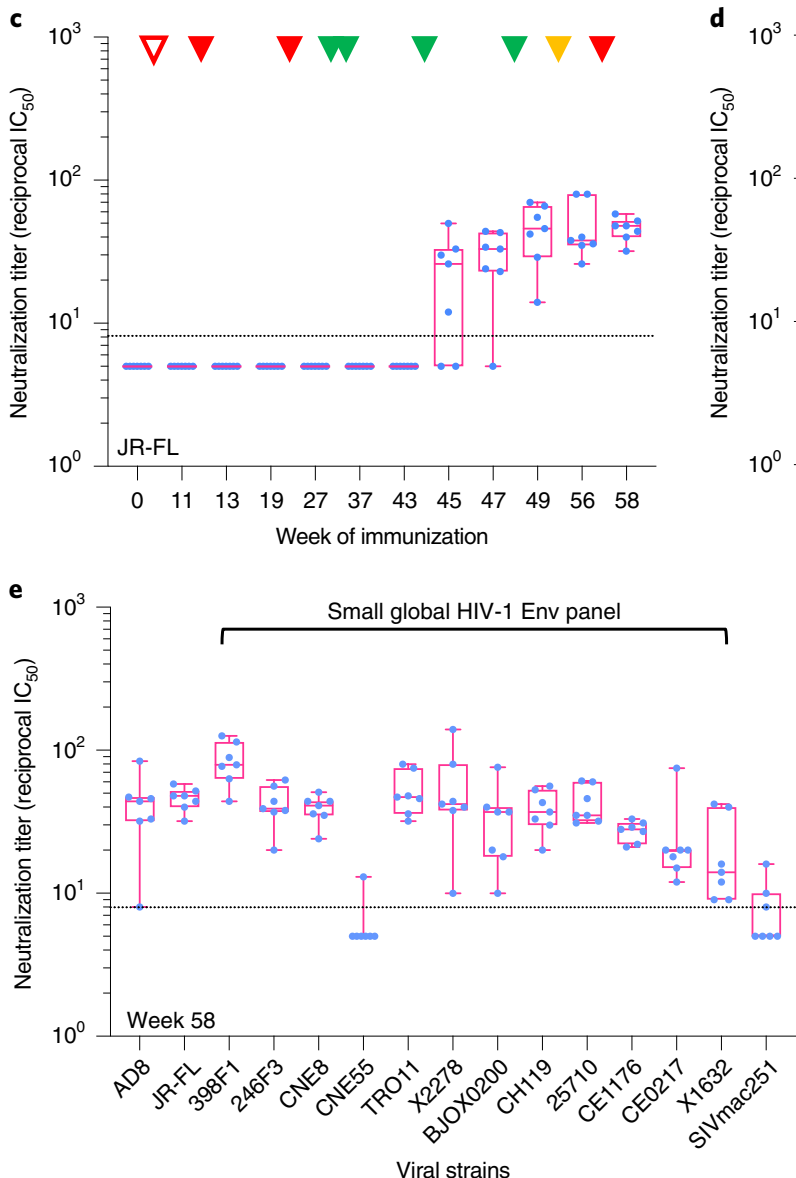

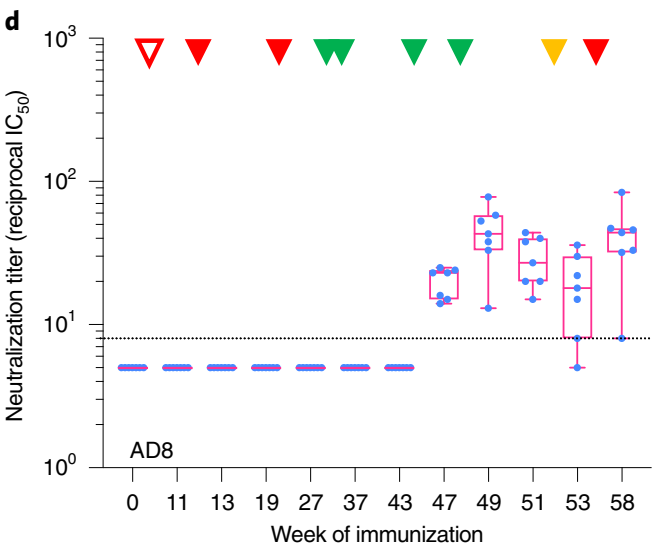

Immunizations:

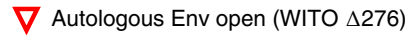

$\nabla$ Autologous Env closed (WITO)

$\nabla$ Heterologous Envs (BG505 + DU422)

7 Heterologous Env (JR-FL)

Fig. 4 | Induction of neutralizing antibodies in immunized macaques over time. a, Serum titers of neutralizing antibodies $\left(I C_{50}\right)$ against the autologous Env (WITO4160.27) in all seven immunized macaques over time, as assayed using pseudoviruses on TZM-bl cells. The data are given in box-and-whisker representation, with the ends of the box representing the first and third quartiles, the horizontal line representing the median, and the whiskers representing the minimum and maximum, with the blue dots representing $I \mathrm{C}_{50}$ for individual macaques. $\mathbf{b}$, Effect of the autologous $\mathrm{V} 3$ peptide (WITO pV3) on autologous neutralization at two time points (week 29 and 37). The assays were performed by incubating the linear $V 3$ peptide with macaque sera prior to addition to the pseudovirus stock. The neutralization titers were moderately reduced by incubation with the $V 3$ peptide but the difference was statistically significant only at week 29 (two-tailed paired $t$-test). c, Serum titers of neutralizing antibodies against a heterologous Env (JR-FL). d, Serum titers of neutralizing antibodies against a heterologous Env (AD8-EO). e, Serum titers of neutralizing antibodies against a panel of heterologous tier-2 Envs at week 58, prior to the start of the live virus challenge. The sequence and timing of immunizations are indicated by the color-coded triangles on the top. Each serum was tested in duplicate wells. Infection controls in the absence of serum were tested in six replicate wells.

The titers became more durable after the third immunization and received the strongest boost by the first heterologous immunization (week 27). To investigate to what extent these neutralizing antibodies were directed against the V3 loop, we tested an autologous V3 peptide (WITO pV3) as a competitor. V3-specific antibodies constituted only a fraction of the total neutralizing antibodies, particularly at the later time point tested (week 37), when the difference between neutralization in the presence and absence of $\mathrm{pV} 3$ was not 
a

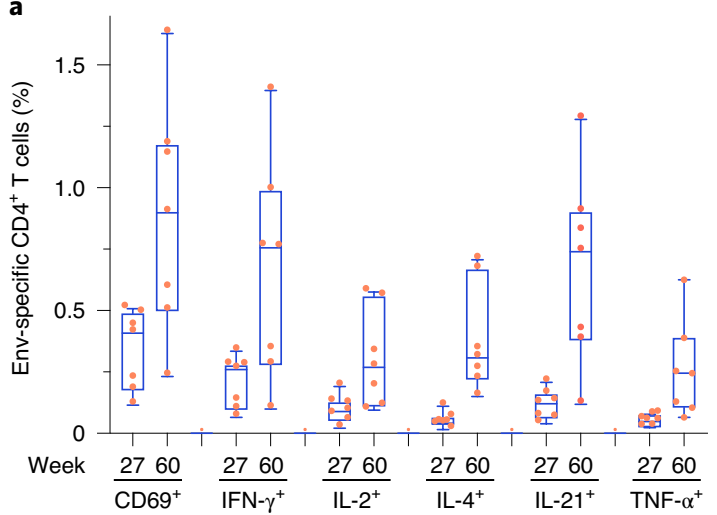

b

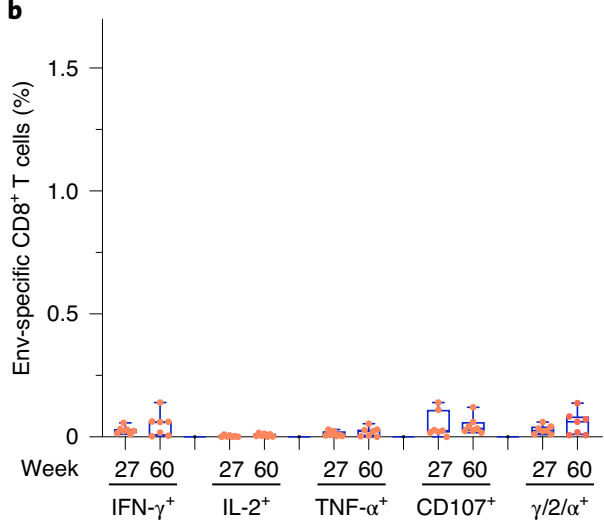

c

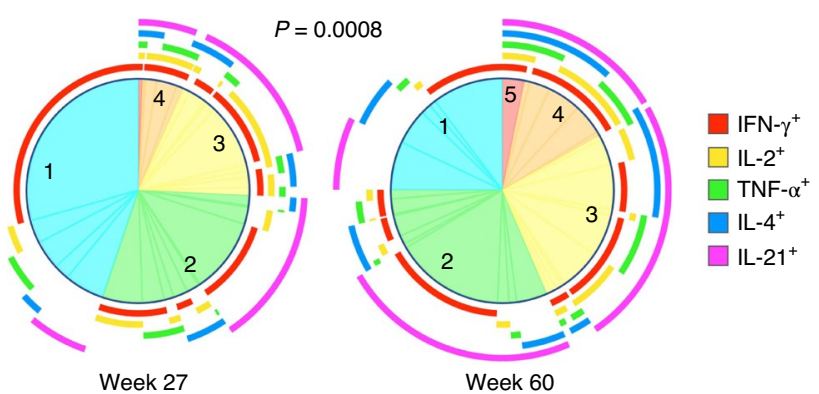

d
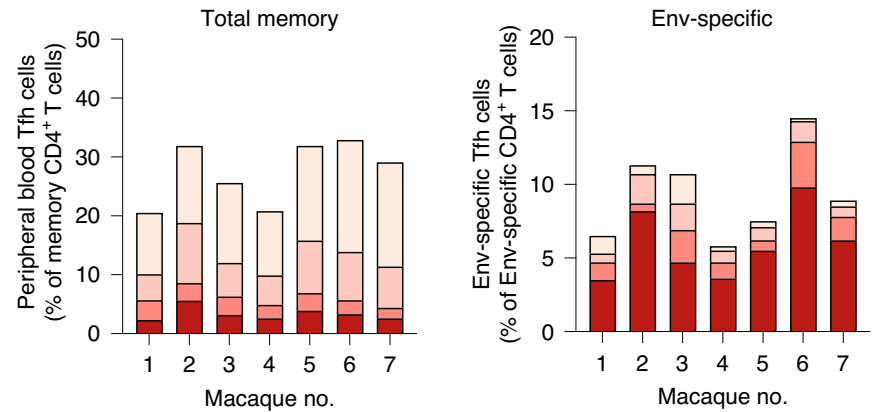

- $\mathrm{CXCR}^{+} \mathrm{CXCR} 3^{+} \mathrm{PD} 1^{+}$ a $\mathrm{CXCR} 5^{+} \mathrm{CXCR} 3^{-} \mathrm{PD} 1^{+}$ 口 $\mathrm{CXCR} 5^{+} \mathrm{CXCR} 3^{+} \mathrm{PD} 1^{-}$ $\square \mathrm{CXCR}^{+} \mathrm{CXCR}^{-} \mathrm{PD} 1^{-}$

Fig. 5 | Induction of Env-specific T cell responses in immunized macaques. The frequency of HIV-1 Env-specific T cells was tested on PBMCs from all seven immunized macaques at week 27 and week 60 of immunization, after ex vivo stimulation with a pool of overlapping HIV-1 clade-B Env peptides. Responses were evaluated by cell-surface staining for different markers and intracellular staining for cytokine production. a, Anti-HIV-1 Env CD4+ T cell responses, calculated as fractions of total memory CD4+ $T$ cells. The cells shown in the chart were double positive for CD154 and the indicated intracellular cytokines or CD69. The data are given in box-and-whisker representation, with the ends of the box representing the first and third quartiles, the horizontal line representing the median, and the whiskers representing the minimum and maximum, with the red dots representing the frequency of Env-specific cells for individual macaques. b. Anti-HIV-1 Env CD8 $8^{+}$T cell responses, calculated as fractions of total memory CD8 ${ }^{+}$T cells. All the cells shown in the chart were double positive for CD69 and the indicated intracellular cytokines or CD107. The last column $(\gamma / 2 / \alpha)$ denotes the total fraction of CD8 ${ }^{+}$T cells expressing at least one of three markers: IFN- $\gamma$, IL-2 or TNF- $\alpha . \mathbf{c}$, Analysis of polyfunctionality in memory CD4+ T cell responses to HIV-1 Env peptides at week 27 and week 60 as conducted using SPICE. The pie chart slices indicate the fraction of Env-specific CD4+ T cells that produce one, two, three, four or five cytokines. The outer arcs, color coded as indicated in the legend, denote the expression of individual cytokines in the underlying slices. A permutation comparison test indicated a significant increase in polyfunctional responses at week 60 compared with week $27(P=0.0008)$. d, Induction of Env-specific circulating Tfh cells in vaccinated macaques. The frequency of various subpopulations of CXCR5 ${ }^{+}$cells (color coded as indicated in the legend) in the peripheral blood of vaccinated macaques at week 60 of immunization is shown for total memory CD4 ${ }^{+} \mathrm{T}$ cells (left) or Env-specific CD4 ${ }^{+}$ T cells (right).

statistically significant (Fig. 4b). No significant differences in autologous neutralization were observed between animals in subgroups 1 and 2, with the exception of higher neutralization titers in subgroup 1 at week 27 and a greater reduction of neutralization by the V3-loop peptide in the same subgroup at week 37 (Extended Data Fig. 3). These results suggest that the protein boosting may have induced slightly higher V3-specific antibody responses.

Heterologous tier-1a neutralizing antibodies, tested against the SF162 strain (clade B), began to appear after the first heterologous immunization and subsequently reached relatively high titers, comparable to those of autologous neutralizing antibodies (Extended Data Fig. 4a). Heterologous tier-1b neutralizing antibodies, tested against the BaL strain (clade B), were also induced at the same time but remained at relatively lower titers throughout the immunization period (Extended Data Fig. 4b), confirming the lack of dominant targeting of tier-1 epitopes by neutralizing antibodies in vaccinated animals. No evidence of heterologous tier-2 neutralization was seen throughout the autologous immunization phase or after the first two heterologous immunizations (Fig. 4c,d). However, after the third heterologous immunization, low titers of bona fide 

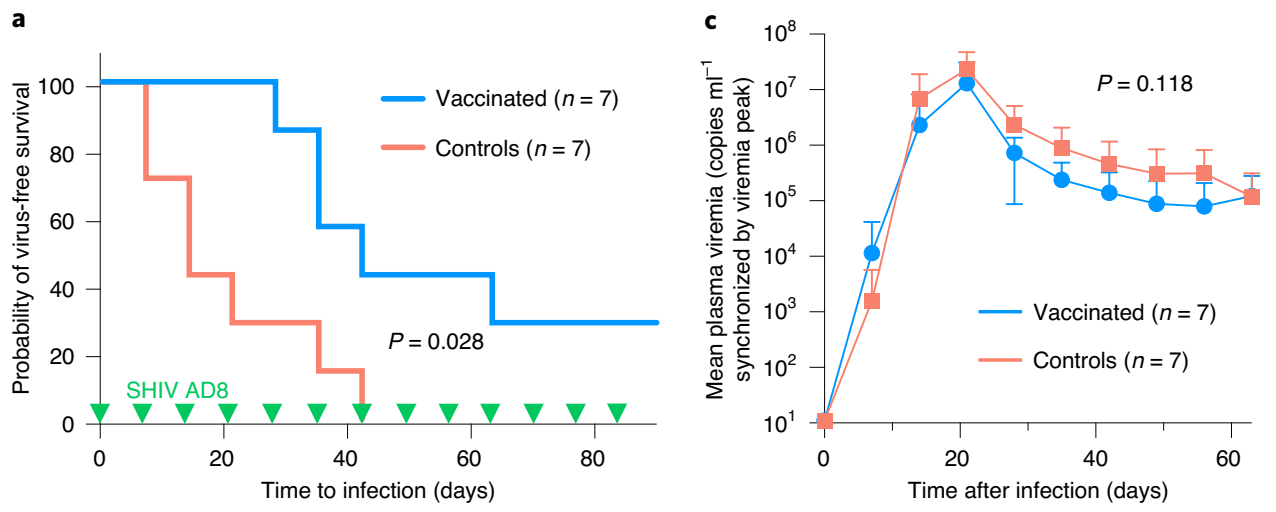

b

\begin{tabular}{|c|c|c|c|c|c|c|}
\hline & \multirow{2}{*}{$\begin{array}{c}\text { Hazard } \\
\text { ratio }\end{array}$} & \multicolumn{2}{|c|}{$95 \% \mathrm{Cl}$} & \multirow{2}{*}{$\begin{array}{l}\text { Per-exposure } \\
\text { risk reduction }\end{array}$} & \multicolumn{2}{|c|}{$95 \% \mathrm{Cl}$} \\
\hline & & Lower & Upper & & Lower & Upper \\
\hline $\begin{array}{l}\text { Vaccinated } \\
\text { versus controls }\end{array}$ & 0.21 & 0.05 & 0.91 & $79 \%$ & $9 \%$ & $95 \%$ \\
\hline
\end{tabular}

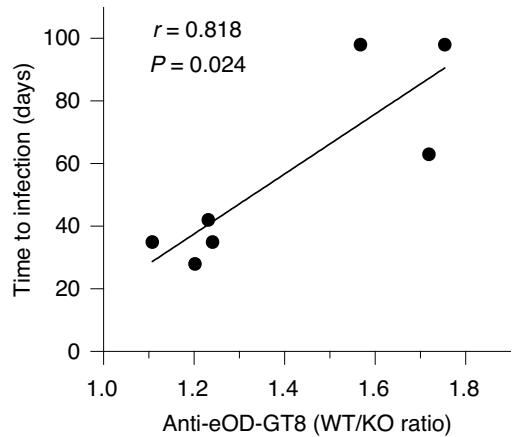

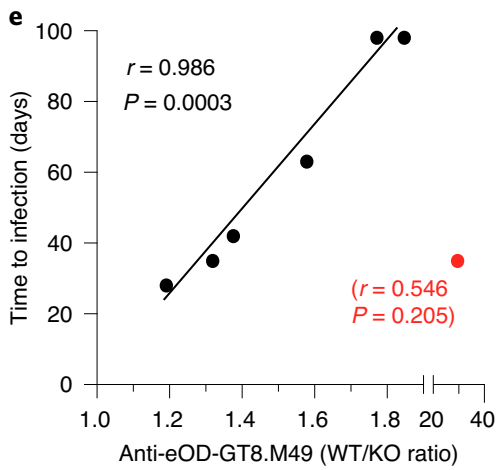

Fig. 6 | Vaccine-induced protection from SHIV AD8 infection and protection correlates in immunized macaques. a, Kaplan-Meier analysis of virus-free survival in the course of 13 weekly intrarectal inoculations of $10 \mathrm{TCID}_{50}$ of the tier-2 heterologous virus SHIV AD8 in seven vaccinated macaques (blue) and seven naive controls (red). Infection was evaluated by the appearance of plasma viremia on two subsequent tests using a sensitive real-time PCR method. Significance was calculated using the Wilcoxon exact test. The green arrows at the bottom denote the 13 sequential weekly mucosal virus inoculations. b. Statistical analysis of hazard ratio and per-exposure risk reduction in vaccinated macaques, using the formula 1 minus the hazard ratio, estimated from a Cox proportional hazards regression using exact partial likelihood with group (vaccinated versus controls) as the regressor. c, Mean level of viremia ( \pm s.d.) in the five vaccinated macaques that became infected (blue) and seven naive controls (red) synchronized by peak of viremia. Levels of viremia were evaluated using quantitative real-time PCR. The courses of viremia in different animals were aligned by peak of viremia (positioned at day 21 for each animal in the graph). Triplicate PCR reactions were run for each serum. $\mathbf{d}, \mathbf{e}$, Correlation between protection (time to infection) and levels of serum antibodies against the CD4-BS, as assessed using an engineered gp120 outer domain probe (eOD-GT8) (d) and a glycan-masked eOD-GT8 probe (mutant 49, M49) (e). A mutated CD4-BS knock-out (KO) probe was used in parallel for subtraction. Each serum was tested in duplicate wells. The correlation was determined using the Spearman test. In e the correlation becomes significant after exclusion of a single outlier (macaque V3, highlighted in red). The correlation coefficient and $P$ value with the inclusion of the outlier are indicated in red. WT, wild-type.

tier-2 heterologous neutralizing antibodies started to appear in all animals, as tested against two difficult-to-neutralize tier- 2 strains, JR-FL (Fig. 4c) and AD8-EO (Fig. 4d), which have Env sequences unrelated to those used for immunization. Heterologous neutralization was boosted by subsequent immunizations although the titers remained relatively low (range of the mean reciprocal $\mathrm{IC}_{50}$, 30-48 against JR-FL and 20-45 against AD8). Of note, the last three immunizations (added as a protocol amendment) did not significantly boost heterologous neutralization (Fig. 4c). Remarkably, by week 58 all the animals had developed broadly tier- 2 neutralizing antibodies that were effective against 11 of $12 \mathrm{HIV}-1$ strains included in a reference global panel of Envs of different geographic and genetic origin ${ }^{56}$ (Fig. 4e). Although four isolates in this panel possess an intermediate neutralization phenotype (tier-1/2) due to increased exposure of the V3 loop ${ }^{57}$, these isolates were not neutralized more efficiently than the others. No significant neutralization was detected against one of the panel isolates (CNE55) or against the irrelevant SIVmac251 Env used as a control. No significant differences in heterologous neutralization were observed when animals in subgroups 1 and 2 were separately analyzed, with the only exception being HIV-1 BaL neutralization at week 49 (Extended Data Fig. 4).

Viral antigen-specific Tcell responses. Virus-specific T cell responses were tested against the two viral antigens expressed by the vaccine, that is, HIV-1 Env and SIV Gag, at two time points: week 27 , after completion of the autologous phase, and week 60 , after completion of the entire immunization protocol. Polyfunctional Env-specific $\mathrm{CD}^{+}{ }^{+} \mathrm{T}$ cell responses were already detectable at week 27 but were markedly boosted in all animals at week 60 (Fig. 5a and Supplementary Table 8). Of note, there was a significant increase in polyfunctionality in $\mathrm{CD}^{+}$T cells at week $60(P=0.0008)($ Fig. $5 c)$, with nearly $50 \%$ of the responding cells co-expressing three cytokines or more, consistent with the elicitation of a strong $\mathrm{T}$ cell helper 
activity for antibody production. Env-specific $\mathrm{CD} 8^{+} \mathrm{T}$ cell responses were also detected, but at much lower levels than the $\mathrm{CD} 4^{+} \mathrm{T}$ cell responses (Fig. 5b). In contrast, SIV Gag-specific CD4 ${ }^{+}$and $\mathrm{CD}^{+}$ T cell responses were low to undetectable at both week 27 and week 60 ((Extended Data Fig. 5a,b). There were no significant differences at either time point between $\mathrm{T}$ cell responses elicited in vaccine subgroups 1 and 2 (Supplementary Table 8).

With regard to $\mathrm{CD} 4^{+}$T cells, we evaluated the frequency and phenotype of circulating T-follicular helper (Tfh) cells given that they represent a key subpopulation in germinal centers for the generation of neutralizing antibody responses ${ }^{58}$. CD $4^{+}$T cells expressing CXCR5 (CD185), a Tfh-associated chemokine receptor that enables access to germinal centers in lymphoid organs, were abundant in the peripheral blood of vaccinated macaques at both week 27 and week 60 , but the majority were negative for the Tfh marker PD-1 (CD279) (Fig. 5d). However, analysis of Env-specific CD4 ${ }^{+}$ T cells showed a skewed phenotypic distribution of CXCR5 ${ }^{+} \mathrm{T}$ cells (CXCR3 $^{+}$or $\mathrm{CXCR}^{-}$), with a dominance of PD-1-positive Tfh cells (Fig. 5 d). The mean frequency of Env-specific CXCR5 ${ }^{+}$cells co-expressing PD- 1 at weeks 27 and 60 was $65.9 \%$ (range, 38.5-80.5) and $77.9 \%$ (range, 63.3-86.9), respectively, with a higher proportion of triple-positive cells (CXCR $5^{+} \mathrm{CXCR} 3^{+} \mathrm{PD}-1^{+}$) compared with all the other CXCR5 ${ }^{+}$subsets. Accordingly, vaccinated macaques had a high frequency of Env-specific $\mathrm{CD} 4^{+}$T cells secreting interleukin (IL)-4 and IL-21, two cytokines that constitute the functional signature of Tfh cells ${ }^{58}$.

Protection from mucosal infection with a heterologous tier-2 SHIV. Having verified the induction of heterologous tier-2 neutralizing antibodies in all immunized animals, we proceeded with the live virus challenge phase (Fig. 2a) using a heterologous tier-2 SHIV strain (SHIV AD8-EO) ${ }^{59}$. Starting at week 60 , all the animals were challenged weekly with intrarectal inoculations of SHIV AD8-EO at $10 \mathrm{TCID}_{50}$ (half-maximal tissue culture infectious doses; equivalent to 0.27 half-maximal macaque infectious $\operatorname{doses}^{60}$ ) for a total of 13 sequential challenges. The non-immunized control group was infected in parallel. Infection was monitored using a sensitive quantitative real-time polymerase chain reaction (PCR) assay for plasma viremia. Control macaques were readily infected, with a restricted mean infection-free time of 3.0 weeks (95\% confidence interval (CI): $1.5-4.5$ weeks), which matches previous results obtained using the same SHIV stock, dose and route ${ }^{61}$. In contrast, two of seven immunized animals never showed any signs of infection after the completion of all 13 weekly inoculations, while the others were infected with a significant delay, with an overall restricted mean infection-free time of 8.1 weeks (95\% CI: $5.2-11.0$ weeks; $P=0.002$ ). On Kaplan-Meier survival analysis there was a significant difference in virus-free survival between vaccinated and control animals $(P=0.028$, Wilcoxon exact test) (Fig. 6a), with a per-exposure risk reduction of $79 \%$ (95\% CI: 9-95\%) (Fig. 6b). No significant difference in virus-free survival was seen between animals in subgroups 1 and 2 , with respective per-exposure risk reductions of $76 \%$ and $86 \%$ (Extended Data Fig. 6a,b). Once infection was detected, the levels of viremia (peak and area under the curve) did not significantly differ between the vaccinated animals and controls (Fig. $6 \mathrm{c}$ ), or between the two vaccine subgroups (Extended Data Fig. 6c).

Assessment of potential protection correlates. The observed protective effect of the vaccine prompted us to investigate the potential immunological correlates of protection, which might shed light on the mechanisms of vaccine efficacy. The binding specificity of vaccine-elicited antibodies was mapped by competition with a panel of bNAbs directed against different supersites of HIV-1 vulnerability. A variety of antibody specificities was seen, as shown by competition with sCD4 and bNAbs against the CD4-BS (VRC01, N6 and 3BNC117), the V3-base glycan region (PGT121 and PGT135), the
V2-apex glycan region (PG16 and PGT145) and the gp120-gp41 interface $(35 \mathrm{O} 22)$, while the fusion peptide region recognized by bNAb VRC34 was not significantly targeted (Extended Data Fig. 7a). To evaluate the elicitation of off-target antibodies, we performed competition assays with a set of well-characterized antibodies against the trimer base, different glycan holes $(289,465,611)$ and the highly variable V1 region of the BG505 Env ${ }^{62,63}$ (Extended Data Fig. 7b). Low levels of competition were detected, in contrast to the results with bNAb epitopes, suggesting that our vaccine did not elicit dominant antibody responses against these irrelevant epitopes. As expected, antibodies against the trimer base were more abundant in animals that received more protein immunizations with SOSIP trimers (subgroup 1), but still remained at relatively low levels.

In a representative protected animal (macaque V3, subgroup 1) we also performed polyclonal epitope mapping by NSEM using a previously reported method ${ }^{64}$. A large fraction of trimers were bound to antigen-binding antibody fragments (Fabs) (9.8\% of total trimers), which appeared to be targeting at least five major regions: a region deep in the inter-protomer groove, compatible with the CD4-BS; a second region in the inter-protomer groove more distal from the trimer axis; a third region at the trimer apex targeted by two Fabs simultaneously; a fourth region at the trimer apex targeted by a single Fab, compatible with the V2-glycan supersite; and a fifth region at the trimer base, as widely reported after SOSIP immunization ${ }^{18-21}$ (Extended Data Fig. 8). These results confirmed that the vaccine was able to elicit antibodies against different trimer regions and that the trimer base was not a dominant target of such antibodies.

Next, we tested the correlations between the number of viral challenges necessary to transmit the infection in individual animals (time to infection) and the antiviral immune responses. On Spearman correlation testing, no significant correlations were observed between protection and antibody titers against SIV Gag p27 or against both autologous (WITO) and heterologous (AD8) Env trimers (Supplementary Table 6). Likewise, serum neutralization titers measured at week 58 against any individual HIV-1 strain, including AD8, were not correlated with protection (Supplementary Table 7). Also, no significant correlations were found between protection and $\mathrm{CD}^{+}$and $\mathrm{CD}^{+} \mathrm{T}$ cell responses or specific phenotypic or functional subpopulations, including Tfh cells (Supplementary Table 8). Only the proportion of Env-specific CXCR $5^{+}$CXCR3-PD- $1^{+}$ Tfh cells at week 60 was significantly correlated with protection after exclusion of a single outlier (Extended Data Fig. 5c).

Next, we tested the reactivity of week 58 immune sera with various epitope-specific probes. No correlations were seen with antibodies against either autologous or heterologous V3-loop peptides, nor against a V1V2 scaffolded antigen (gp70), the recognition of which had previously been correlated with a reduced risk of infection in the RV144 clinical trial ${ }^{65}$ (Extended Data Fig. 9). However, a significant correlation was found with antibodies against the CD4-BS, as assayed using an engineered outer domain (OD) probe, eOD-GT8, versus its mutated CD4-BS knock-out counter$\operatorname{part}^{66}(r=0.818 ; P=0.024)$ (Fig. $\left.6 \mathrm{~d}\right)$. Binding to a glycan-masked eOD-GT8-derivative probe (eOD-GT8 M49) ${ }^{67}$ also showed a highly significant correlation $(r=0.986 ; P=0.0003)$ after the removal of a single outlier for which the pre-immune serum already had high baseline reactivity with the probe (Fig. 6e).

To assess the interaction of serum antibodies from protected animals with the native, membrane-expressed Env, we tested their ability to bind to the surface of primary $\mathrm{CD} 4^{+} \mathrm{T}$ cells infected in vitro with SHIV AD8, the same virus used for challenge, and to mediate antibody-dependent cellular cytotoxicity (ADCC). These are biologically relevant assays because they measure antibody recognition of membrane-anchored Env spikes endogenously produced by primary cells. Given that cell-surface binding assays are biased by the bystander effect of uninfected $\mathrm{CD} 4^{+}$cells, which passively adsorb extracellular soluble gp120 through CD4, we used a vali- 
dated method to selectively analyze infected cells, which downregulate CD4 and, therefore, do not passively bind free gp120 (refs ${ }^{68,69}$ ). Both cell-surface binding and ADCC showed a trend toward correlation with protection, which became significant in both cases $(P=0.016$ and 0.028 , respectively) after exclusion of a single outlier (Extended Data Fig. 9d,e). Further corroborating the presence of trimer-binding antibodies specific for the CD4-BS, binding to the surface of primary SHIV AD8-infected cells was significantly reduced by pre-treatment with a Fab of the bNAb VRC01 $(P<0.0001)$ (Extended Data Fig. 9f).

\section{Discussion}

The present study provides a proof of principle that a multiclade HIV-1 env-gag mRNA vaccine is safe and immunogenic, can induce neutralization breadth accompanied by polyfunctional $\mathrm{CD}^{+} \mathrm{T}$ cell responses, and can protect rhesus macaques from infection with a heterologous tier-2 virus. Specifically, the results that we obtained both in mice and macaques validate the further development of a VLP-generating env-gag mRNA platform for the presentation of native HIV-1 Env to the immune system. The use of mRNA as a vehicle may offer significant advantages over immunization with exogenous proteins in terms of the challenges, cost and time required for immunogen manufacturing for human clinical trials. In the specific case of HIV-1, it may also overcome many of the issues that arise with the recombinant production of Env trimer immunogens, especially glycan processing, antigenic modifications associated with trimer stabilization, and the immunodominance of off-target epitopes. Furthermore, many primary HIV-1 Envs that might serve as effective immunogens are not amenable to expression as stabilized soluble trimers.

The vaccine platform that we designed combines a series of unique features, all of which may have contributed to its efficacy. One of the most notable is the co-formulation of mRNAs encoding membrane-anchored Env and Gag proteins, which leads to the endogenous generation of VLPs similar to the viral particles produced during natural infection. Although in vivo-generated VLPs are difficult to visualize, in vitro transfection experiments documented a robust and consistent VLP formation with diverse HIV-1 Env immunogens. The results obtained in mice by direct comparison of co-formulated env-gag mRNA with env mRNA alone provided supporting evidence for the advantages of this design. Other factors that were presumably critical were the initial priming with a transmitted-founder HIV-1 Env lacking the N276 glycan, which is in line with lineage-based vaccine strategies aimed at the early recruitment of germline bNAb precursors, and the intensive multiclade heterologous boosting. Corroborating the importance of such intensive multiclade stimulation, neutralization breadth started to appear only after the third heterologous immunization.

As often occurs in preclinical and clinical vaccine studies, the immunological mechanisms responsible for the risk reduction conferred by our vaccine remain uncertain. However, analysis of protection correlates conducted at the time of virus challenge provided some clues about the potential protective mechanisms. A significant correlation was observed with antibodies against the CD4-BS, a key supersite of HIV-1 vulnerability, which supports a role of the humoral immune response in mediating protection. In rhesus macaques, the lineages of anti-CD4-BS bNAbs are not yet clearly defined and, therefore, it was not possible to test the ability of our priming immunogen to engage germline macaque antibodies against this site. However, we can postulate that removal of the N276 glycan, which effectively shields the CD4-BS from germline $\mathrm{bNAb}$ precursors in humans, may have succeeded in recruiting the relevant macaque $\mathrm{B}$ cell precursors at the onset of the immunization protocol. The multiple booster immunizations with closed, tier-2 Envs from three different clades may have then focused the B cell responses on shared tier- 2 epitopes, thereby fostering the maturation of broad-spectrum neutralizing antibodies. Consistent with a role of humoral immune responses in protection, our vaccine induced robust polyfunctional Env-specific CD4 ${ }^{+} \mathrm{T}$ cells at the time of virus challenge. As previously reported with mRNA vaccines $^{41,70-72}$, we also documented the induction of virus-specific Tfh cells, a key subpopulation that was shown to support B cell differentiation into affinity-matured long-lived plasma cells and promote bNAb development against HIV-1 (ref. ${ }^{73}$ ).

Despite these encouraging results, there are some limitations with the present study. First, the neutralization titers elicited by our vaccine against the challenge virus, $\mathrm{AD} 8$, and other heterologous tier-2 viruses were relatively low and did not reach levels that have previously been reported to be protective in vivo ${ }^{7,74-78}$. This suggests that protection was unlikely to be due to neutralizing antibodies alone, but rather to a combination of neutralizing antibodies with other immunologic mechanisms. One such mechanism could be ADCC, which was effectively documented in our immunized animals. Moreover, the induction of antibodies against multiple Env antigenic sites by our vaccine raises the possibility of a cooperative effect between antibodies with different specificity and functionality. Another limitation of our study was that the protective effect of the vaccine was partial, with only two animals being fully protected. However, it should be emphasized that we used a heterologous tier-2 strain (SHIV AD8) that is particularly difficult to neutralize, and still the vaccine induced a $79 \%$ per-exposure risk reduction upon 13 sequential mucosal challenges, a protocol that best approximates the prevalent modality of sexual transmission in humans. Given that mucosal transmission of HIV-1 in humans is an inefficient event, this level of risk reduction might still have a significant impact on viral transmission. Finally, the vaccine has so far been tested only in a single study, and therefore the results need to be confirmed with additional preclinical and clinical trials.

In conclusion, the env-gag VLP mRNA platform described here offers a promising approach for the development of a preventive HIV-1 vaccine. Although the intensive immunization schedule with high mRNA doses that we used was remarkably well tolerated, a vaccination regimen encompassing seven or more sequential immunizations would be difficult to implement in humans. Refinements of the current protocol, including improvements in both the yield and quality of VLP generation, may increase the efficacy of immunization and thereby require fewer vaccine boosts. Specific modifications in the choice of Env immunogens and mRNA formulations are being evaluated, in parallel with the original protocol, in a second vaccine-challenge study in rhesus macaques that is currently under way.

\section{Online content}

Any methods, additional references, Nature Research reporting summaries, source data, extended data, supplementary information, acknowledgements, peer review information; details of author contributions and competing interests; and statements of data and code availability are available at https://doi.org/10.1038/ s41591-021-01574-5.

Received: 9 June 2021; Accepted: 6 October 2021; Published online: 9 December 2021

\section{References}

1. Mascola, J. R. \& Haynes, B. F. HIV-1 neutralizing antibodies: understanding nature's pathways. Immunol. Rev. 254, 225-244 (2013).

2. Kelsoe, G. \& Haynes, B. F. Host controls of HIV broadly neutralizing antibody development. Immunol. Rev. 275, 79-88 (2017).

3. Burton, D. R. \& Mascola, J. R. Antibody responses to envelope glycoproteins in HIV-1 infection. Nat. Immunol. 16, 571-576 (2015).

4. Haynes, B. F., Burton, D. R. \& Mascola, J. R. Multiple roles for HIV broadly neutralizing antibodies. Sci. Transl. Med. 11, eaaz2686 (2019).

5. Burton, D. R. \& Hangartner, L. Broadly neutralizing antibodies to HIV and their role in vaccine design. Annu. Rev. Immunol. 34, 635-659 (2016). 
6. Excler, J. L. \& Michael, N. L. Lessons from HIV-1 vaccine efficacy trials. Curr. Opin. HIV AIDS 11, 607-613 (2016).

7. Hessell, A. J., Malherbe, D. C. \& Haigwood, N. L. Passive and active antibody studies in primates to inform HIV vaccines. Expert Rev. Vaccines 17, 127-144 (2018).

8. Corey, L. et al. Immune correlates of vaccine protection against HIV-1 acquisition. Sci. Transl. Med. 7, 310rv317 (2015).

9. Rerks-Ngarm, S. et al. Vaccination with ALVAC and AIDSVAX to prevent HIV-1 infection in Thailand. N. Engl. J. Med. 361, 2209-2220 (2009).

10. Cohen, J. Combo of two HIV vaccines fails its big test. Science 367, 611-612 (2020)

11. Easterhoff, D. et al. HIV vaccine delayed boosting increases Env variable region 2-specific antibody effector functions. JCI Insight 5, e131437 (2020).

12. Sanders, R. W. et al. A next-generation cleaved, soluble HIV-1 Env trimer, BG505 SOSIP.664 gp140, expresses multiple epitopes for broadly neutralizing but not non-neutralizing antibodies. PLoS Pathog. 9, e1003618 (2013).

13. Kwon, Y. D. et al. Crystal structure, conformational fixation and entry-related interactions of mature ligand-free HIV-1 Env. Nat. Struct. Mol. Biol. 22, 522-531 (2015).

14. de Taeye, S. W. et al. Immunogenicity of stabilized HIV-1 envelope trimers with reduced exposure of non-neutralizing epitopes. Cell 163, 1702-1715 (2015)

15. Sullivan, J. T. et al. High-throughput protein engineering improves the antigenicity and stability of soluble HIV-1 envelope glycoprotein SOSIP trimers. J. Virol. 91, e00862-17 (2017).

16. Torrents de la Peña, A. et al. Improving the immunogenicity of native-like HIV-1 envelope trimers by hyperstabilization. Cell Rep. 20, 1805-1817 (2017)

17. Zhang, P. et al. Interdomain stabilization impairs CD4 binding and improves immunogenicity of the HIV-1 envelope trimer. Cell Host Microbe 23, 832-844 (2018)

18. Sanders, R. W. et al. HIV-1 neutralizing antibodies induced by native-like envelope trimers. Science 349, aac4223 (2015).

19. Klasse, P. J. et al. Sequential and simultaneous immunization of rabbits with HIV-1 envelope glycoprotein SOSIP.664 trimers from clades A, B and C. PLoS Pathog. 12, e1005864 (2016).

20. Hu, J. K. et al. Murine antibody responses to cleaved soluble HIV-1 envelope trimers are highly restricted in specificity. J. Virol. 89, 10383-10398 (2015).

21. Zhou, T. et al. Quantification of the impact of the HIV-1-glycan shield on antibody elicitation. Cell Rep. 19, 719-732 (2017).

22. Pauthner, M. et al. Elicitation of robust tier 2 neutralizing antibody responses in nonhuman primates by HIV envelope trimer immunization using optimized approaches. Immunity 46, 1073-1088 (2017)

23. McCoy, L. E. et al. Holes in the glycan shield of the native HIV envelope are a target of trimer-elicited neutralizing antibodies. Cell Rep. 16, 2327-2338 (2016)

24. Kwong, P. \& Mascola, J. P. HIV-1 vaccines based on antibody identification, B cell ontogeny and epitope structure. Immunity 48, 855-871 (2018).

25. Kwong, P. D., DeKosky, B. J. \& Ulmer, J. B. Antibody-guided structure-based vaccines. Semin. Immunol. 50, 101428 (2020).

26. $\mathrm{Xu}, \mathrm{K}$. et al. Epitope-based vaccine design yields fusion peptide-directed antibodies that neutralize diverse strains of HIV-1. Nat. Med. 24, 857-867 (2018).

27. Kong, R. et al. Antibody lineages with vaccine-induced antigen-binding hotspots develop broad HIV neutralization. Cell 178, 567-584 (2019).

28. Steichen, J. M. et al. A generalized HIV vaccine design strategy for priming of broadly neutralizing antibody responses. Science 366, eaax4380 (2019).

29. Saunders, K. O. et al. Targeted selection of HIV-specific antibody mutations by engineering Bcell maturation. Science 366, eaay7199 (2019).

30. Chen, X. et al. Vaccination induces maturation in a mouse model of diverse unmutated VRC01-class precursors to HIV-neutralizing antibodies with $>50 \%$ breadth. Immunity 54, 324-339 (2021)

31. Bradley, T. et al. Pentavalent HIV-1 vaccine protects against simian-human immunodeficiency virus challenge. Nat. Commun. 8, 15711 (2017).

32. Barouch, D. H. et al. Evaluation of a mosaic HIV-1 vaccine in a multicentre, randomised, double-blind, placebo-controlled, phase 1/2a clinical trial (APPROACH) and in rhesus monkeys (NHP 13-19). Lancet 392, 232-243 (2018).

33. Felber, B. K. et al. Co-immunization of DNA and protein in the same anatomical sites induces superior protective immune responses against SHIV challenge. Cell Rep. 31, 107624 (2020).

34. Om, K. et al. Adjuvanted HIV-1 vaccine promotes antibody-dependent phagocytic responses and protects against heterologous SHIV challenge. PLoS Pathog. 16, e1008764 (2020).

35. Arunachalam, P. S. et al. T cell-inducing vaccine durably prevents mucosal SHIV infection even with lower neutralizing antibody titers. Nat. Med. 26 932-940 (2020).

36. Pardi, N. et al. Characterization of HIV-1 nucleoside-modified mRNA vaccines in rabbits and rhesus macaques. Mol. Ther. Nucleic Acids 15, 36-47 (2019).

37. Moyo, N. et al. Efficient induction of T cells against conserved HIV-1 regions by mosaic vaccines delivered as self-amplifying mRNA. Mol. Ther. Methods Clin. Dev. 12, 32-46 (2018).
38. Saunders, K. O. et al. Lipid nanoparticle encapsulated nucleoside-modified mRNA vaccines elicit polyfunctional HIV-1 antibodies comparable to proteins in nonhuman primates. NPJ Vaccines 6, 50 (2021).

39. Pardi, N., Hogan, M. J., Porter, F. W. \& Weissman, D. mRNA vaccines: a new era in vaccinology. Nat. Rev. Drug Discov. 17, 261-279 (2018).

40. Alameh, M.-G., Weissman, D. \& Pardi, N. Messenger RNA-based vaccines against infectious diseases. Curr. Top. Microbiol. Immunol. https://doi. org/10.1007/82_2020_202 (2020).

41. Corbett, K. S. et al. Evaluation of the mRNA-1273 vaccine against SARS-CoV-2 in nonhuman primates. N. Engl. J. Med. 383, 1544-1555 (2020).

42. Polack, F. P. et al. Safety and efficacy of the BNT162b2 mRNA Covid-19 vaccine. N. Engl. J. Med. 383, 2603-2615 (2020).

43. Baden, L. R. et al. Efficacy and safety of the mRNA-1273 SARS-CoV-2 vaccine. N. Engl. J. Med. 384, 403-416 (2021).

44. Manolova, V. et al. Nanoparticles target distinct dendritic cell populations according to their size. Eur. J. Immunol. 38, 1404-1413 (2008).

45. Chen, C. W., Saubi, N. \& Joseph-Munne, J. Design concepts of virus-like particle-based HIV-1 vaccines. Front. Immunol. 11, 573157 (2020).

46. Andersson, A. C., Schwerdtfeger, M. \& Holst, P. J. Virus-like-vaccines against HIV. Vaccines 6, 10 (2018).

47. Effio, C. L. \& Hubbuch, J. Next generation vaccines and vectors: designing downstream processes for recombinant protein-based virus-like particles. Biotechnol. J. 10, 715-727 (2015).

48. Buonaguro, L. et al. High efficient production of Pr55(gag) virus-like particles expressing multiple HIV-1 epitopes, including a gp120 protein derived from an Ugandan HIV-1 isolate of subtype A. Antiviral Res. 49 35-47 (2001).

49. Gheysen, D. et al. Assembly and release of HIV-1 precursor Pr55gag virus-like particles from recombinant baculovirus-infected insect cells. Cell 59, 103-112 (1989).

50. Haffar, O. et al. Human immunodeficiency virus-like, nonreplicating, gag-env particles assemble in a recombinant vaccinia virus expression system. J. Virol. 64, 2653-2659 (1990).

51. Zhu, P. et al. Distribution and three-dimensional structure of AIDS virus envelope spikes. Nature 441, 847-852 (2006).

52. Keele, B. F. et al. Identification and characterization of transmitted and early founder virus envelopes in primary HIV-1 infection. Proc. Natl Acad. Sci. USA 105, 7552-7557 (2008).

53. Voss, J. E. et al. Elicitation of neutralizing antibodies targeting the V2 apex of the HIV envelope trimer in a wild-type animal model. Cell Rep. 21, 222-235 (2017)

54. Kothe, D. L. et al. Antigenicity and immunogenicity of HIV-1 consensus subtype B envelope glycoproteins. Virology 360, 218-234 (2007).

55. Li, H. et al. Envelope residue 375 substitutions in simian-human immunodeficiency viruses enhance CD4 binding and replication in rhesus macaques. Proc. Natl Acad. Sci. USA 113, 3413-3422 (2016).

56. deCamp, A. et al. Global panel of HIV-1 Env reference strains for standardized assessments of vaccine-elicited neutralizing antibodies. J. Virol. 88, 2489-2507 (2014).

57. Han, Q. et al. Difficult-to-neutralize global HIV-1 isolates are neutralized by antibodies targeting open envelope conformations. Nat. Commun. 10, 2898 (2019).

58. Crotty, S. Follicular helper CD4 T cells (TFH). Annu. Rev. Immunol. 29, 621-663 (2011).

59. Gautam, R. et al. Pathogenicity and mucosal transmissibility of the R5-tropic simian/human immunodeficiency virus SHIV(AD8) in rhesus macaques: implications for use in vaccine studies. J. Virol. 86, 8516-8526 (2012)

60. Gautam, R. et al. A single injection of anti-HIV-1 antibodies protects against repeated SHIV challenges. Nature 533, 105-109 (2016).

61. Gautam, R. et al. A single injection of crystallizable fragment domain-modified antibodies elicits durable protection from SHIV infection. Nat. Med. 24, 610-616 (2018).

62. Cottrell, C. A. et al. Mapping the immunogenic landscape of near-native HIV-1 envelope trimers in non-human primates. PLoS Pathog. 16, e1008753 (2020)

63. van Schooten, J. et al. Antibody responses induced by SHIV infection are more focused than those induced by soluble native HIV-1 envelope trimers in non-human primates. PLoS Pathog. 17, e1009736 (2021).

64. Nogal, B. et al. Mapping polyclonal antibody responses in non-human primates vaccinated with HIV Env trimer subunit vaccines. Cell Rep. 30, 3755-3765 (2020).

65. Haynes, B. F. et al. Immune-correlates analysis of an HIV-1 vaccine efficacy trial. N. Engl. J. Med. 366, 1275-1286 (2012)

66. Jardine, J. G. et al. HIV-1 broadly neutralizing antibody precursor B cells revealed by germline-targeting immunogen. Science 351, 1458-1463 (2016)

67. Duan, H. et al. Glycan masking focuses immune responses to the HIV-1 CD4-binding site and enhances elicitation of VRC01-class precursor antibodies. Immunity 49, 301-311 (2018). 
68. Prevost, J. et al. Incomplete downregulation of CD4 expression affects HIV-1 Env conformation and antibody-dependent cellular cytotoxicity responses. J. Virol. 92, e00484-18 (2018)

69. Richard, J. et al. Uninfected bystander cells impact the measurement of HIV-specific antibody-dependent cellular cytotoxicity responses. $m$ Bio 9 , e00358-18 (2018).

70. Pardi, N. et al. Nucleoside-modified mRNA vaccines induce potent T follicular helper and germinal center B cell responses. J. Exp. Med. 215, 1571-1588 (2018).

71. Garrido, C. et al. SARS-CoV-2 vaccines elicit durable immune responses in infant rhesus macaques. Sci. Immunol. 6, eabj3684 (2021).

72. Painter, M. M. et al. Rapid induction of antigen-specific CD4(+) T cells is associated with coordinated humoral and cellular immunity to SARS-CoV-2 mRNA vaccination. Immunity 54, 2133-2142 (2021).

73. Locci, M. et al. Human circulating PD-1+CXCR3-CXCR5+ memory Tfh cells are highly functional and correlate with broadly neutralizing HIV antibody responses. Immunity 39, 758-769 (2013).

74. Moldt, B. et al. Highly potent HIV-specific antibody neutralization in vitro translates into effective protection against mucosal SHIV challenge in vivo. Proc. Natl Acad. Sci. USA 109, 18921-18925 (2012).
75. Montefiori, D. C. \& Mascola, J. R. Neutralizing antibodies against HIV-1: can we elicit them with vaccines and how much do we need? Curr. Opin. HIV AIDS 4, 347-351 (2009).

76. Parren, P. W. et al. Antibody protects macaques against vaginal challenge with a pathogenic R5 simian/human immunodeficiency virus at serum levels giving complete neutralization in vitro. J. Virol. 75, 8340-8347 (2001)

77. Shingai, M. et al. Passive transfer of modest titers of potent and broadly neutralizing anti-HIV monoclonal antibodies block SHIV infection in macaques. J. Exp. Med. 211, 2061-2074 (2014).

78. Pauthner, M. G. et al. Vaccine-induced protection from homologous tier 2 SHIV challenge in nonhuman primates depends on serum-neutralizing antibody titers. Immunity 50, 241-252 (2019).

Publisher's note Springer Nature remains neutral with regard to jurisdictional claims in published maps and institutional affiliations.

This is a U.S. government work and not under copyright protection in the U.S.; foreign copyright protection may apply 2021 


\section{Methods}

Animals. BALB/c mice (6-8 weeks old) were obtained from Charles River. The experiments in mice were carried out in compliance with all pertinent US National Institutes of Health (NIH) regulations and were approved by the Animal Care and Use Committee (ACUC) of Moderna. Fourteen outbred naive female rhesus macaques of Indian origin, 6-10 years of age, were genotyped and selected as negative for the protective major histocompatibility complex (MHC) class I alleles $M a m u-A^{\star} 01, M a m u-B^{\star} 08$ and $M a m u-B^{\star} 17$. The animals were randomly assigned to the study groups, which were balanced for age, weight, complete blood count and chemistry profiles. All the animals were housed at Bioqual in Rockville, MD, USA according to the guidelines of the Association for Assessment and Accreditation of Laboratory Animal Care standards, with the exception of three vaccine-naive controls, which were housed at the NIH Animal Facility in Poolesville, MD, USA. During the challenge phase, the animals received the same dose of the same viral stock (SHIV AD8-EO) in both facilities and the veterinary staff followed the same operating procedures. The study protocol was approved by both the NIH and the Bioqual ACUCs.

mRNA immunogens. Sequence-optimized mRNAs encoding the immunogens were synthesized in vitro using an optimized T7 RNA polymerase-mediated transcription reaction with $N^{1}$-methylpseudouridine-5'-triphosphate utilized in place of uridine-5'-triphosphate ${ }^{79}$. The in vitro transcription DNA template contained the target open reading frame flanked by 5 ' and 3 ' untranslated regions and an encoded polyA tail. The GenBank accession numbers for the mRNA immunogens are: WITO4160.27.ENV.gp145.N276D.(1-745aa), MZ362872; WITO4160.27.ENV.gp145.G153E.(1-745aa), MZ362873; DU422.1.ENV.K295N.D386N.375Y.(1-745aa), MZ362874; BG505.W6M ENV.C2.T332N.S375Y.gp160, MZ362875; SIVmac239.gag.P55, MZ362876. After transcription, a cap 1 structure was added enzymatically using vaccine capping enzyme and 2'-O-methyltransferase (New England Biolabs). Purified mRNA was sterilized by filtration and co-formulated into the same lipid nanoparticle using a method described previously ${ }^{80}$. In brief, mRNA was diluted in acetate, $\mathrm{pH}$ 5.0, and mixed with lipids (SM-102:1,2-distearoyl-sn-glycero3 -phosphocholine: cholesterol: polyethylene glycol 2000-dimyristoyl glycerol) dissolved in neat ethanol at a ratio of 3:1 (mRNA:lipids). The product was then dialyzed against PBS, $\mathrm{pH} 7.4$, tested for encapsulation, particle size and polydispersity, and mRNA purity and endotoxin, and was deemed acceptable for in vivo use. The env: gag mRNA ratio ranged between 1.7:1 and 2.2:1 in the various immunogen formulations (Supplementary Table 3).

In vitro transfection and VLP capture and quantification. The mRNAs encoding different HIV-1 Env constructs $(1 \mu \mathrm{g})$ and SIVmac239 Gag $(2 \mu \mathrm{g})$ were co-transfected into HEK293T cells. The RNA mixtures were added to 6-well plates $\left(10^{6}\right.$ cells per well) with $6 \mu$ l transfection reagent and transfection booster from the TransIT-mRNA Transfection Kit (Mirus). Supernatants were collected $48 \mathrm{~h}$ after transfection, centrifuged at 1,000 r.p.m. and filtered through a $0.45 \mu \mathrm{M}$ PES filter (Millipore). For VLP capture from culture supernatants, the anti-Env bNAb PG16 and PGT128 were incubated with Protein G-conjugated immunomagnetic beads (Dynabeads; Life Technologies) at $20 \mu \mathrm{g} \mathrm{ml}^{-1}$. Antibody-armed beads were incubated with VLP-containing supernatants at room temperature for $30 \mathrm{~min}$ and then washed three times with PBS containing $0.025 \%$ (vol/vol) casein (Vector Laboratories) to remove the unbound material. Captured VLPs were lysed with RIPA buffer on the beads, and the released p27 Gag protein concentration was measured using an SIV p27 antigen in-house ELISA. Serial dilutions of reference p27 protein and lysed VLP p27 were coated onto 96-well ELISA plates (Corning) at $4{ }^{\circ} \mathrm{C}$ overnight. Then, the plates were blocked with $1 \mathrm{x}$ casein at room temperature for $1 \mathrm{~h}$, followed by the addition of mouse anti-p27 monoclonal antibody (AIDS Reagent Program, cat. no. 3537) at $1 \mu \mathrm{g} \mathrm{ml}^{-1}$. After washing, horseradish peroxidase (HRP)-conjugated goat anti-mouse immunoglobulin G (IgG) (Invitrogen, cat. no. A16072) was added for $1 \mathrm{~h}$ at room temperature. The optical density at $450 \mathrm{~nm}$ was measured. The VLP p 27 concentrations were calculated using a doseresponse curve fit with a 5-parameter non-linear function in GraphPad Prism 8. For concentrating VLPs, $25 \mathrm{ml}$ VLP-containing supernatants were layered onto a $10-20 \%$ linear gradient sucrose PBS buffer and centrifuged at 29,300 r.p.m. (Beckman Rotor Type $50.2 \mathrm{Ti}, \sim 78,000 \times \mathrm{g}$ ) for $2 \mathrm{~h}$ at $4^{\circ} \mathrm{C}$. After centrifugation, the supernatants were discarded and the VLP pellets were resuspended in PBS and stored at $4^{\circ} \mathrm{C}$ for NSEM or gp120/p27 ELISA.

Mouse immunization protocol. BALB/c mice were injected with mRNA formulations in a volume of $0.05 \mathrm{ml}$ using a sterile syringe by intramuscular injection in the hind leg. The animals were immunized at days 0 and 28 with $2.5 \mu$ g HIV-1 env 426c.D276.D460.D463(1-745aa) mRNA alone (group 1; $n=8$ ) or co-formulated with $2.5 \mu \mathrm{g}$ SIVmac239 gag mRNA (group $2 ; n=8$ ). Control mice $(n=4)$ received PBS because previous studies have proven that the mRNA formulations being tested do not create substantial levels of non-specific immunity $^{81}$. Blood was collected on day $-1,14,28,43$ and 57 . Blood collections did not exceed $1 \%$ of the total body weight or $10 \mathrm{ml} \mathrm{kg}^{-1}$ over a 2 week period, per ACUC protocol. Regarding the sample size, a Wilcoxon sum rank test with a two-tailed level of significance of 0.05 would have $>80 \%$ power to detect an effect size of 1.6 between the Env and Env-Gag vaccine arms, each with a sample size of 8 , and to detect an effect size of 2 between a vaccine arm and the control arm, with sample sizes of 8 and 4 , respectively.

Macaque immunization protocol and live virus challenges. Formulated mRNA was diluted in sterile PBS immediately before the immunizations, as required to reach the desired volume. The protein immunization doses were prepared by mixing $100 \mu \mathrm{g}$ SOSIP trimer with $100 \mu \mathrm{l}$ adjuvant (Adjuplex; Sigma) in PBS per animal. The final inoculation volume was $500 \mu \mathrm{l}$ per animal. Animals were vaccinated intramuscularly at week $0,11,19,27,35,43,47,51,56$ and 59 with either mRNA or protein into the right hind leg. At week 27, 35, 43 and 47, the animals received two separate inoculations (DU422 375Y.1-745 and BG505 $375 \mathrm{Y}$ gp160 mRNA or protein), at least 1 inch apart, in the same quadriceps muscle. The mRNA and protein doses inoculated at each time point are listed in Supplementary Table 3. For live virus challenge, molecularly cloned SHIV AD8 previously titrated in vivo was inoculated intrarectally. Animals were scheduled to receive 13 sequential low-dose intrarectal inoculations containing $10 \mathrm{TCID}_{50}$ of SHIV AD8-EO diluted in sterile PBS. For reference, a previous study conducted with $10 \mathrm{TCID}_{50}$ of the same viral stock via intrarectal inoculation in 12 naive macaques yielded infection after a mean of $3.8 \pm 1$.1 inoculations (range, 2-6; median, 3 inoculations) ${ }^{61}$. Challenges were suspended in animals with confirmed infection. All blood samples (serum, plasma or peripheral blood mononuclear cells (PBMCs)) were collected either on the day of or 2 weeks after vaccine inoculations. PBMCs were isolated by Ficoll and frozen in 10\% DMSO and 90\% FBS in CoolCell LX cell freezing containers (Corning), left at $-80^{\circ} \mathrm{C}$ overnight and then transferred to a vapor-phase liquid nitrogen freezer (Thermo Scientific) for long-term storage. Regarding the sample size, with seven rhesus macaques per arm, the study had $>80 \%$ power to detect the protective efficacy of the vaccine against SHIV infection if the protective efficacy, defined as 1 minus the hazard ratio, was not less than 0.90 in a Wilcoxon sum rank test on the time to infection with a two-tailed significance level of 0.05 .

Mutagenesis, protein expression and purification. Mutations were introduced by site-directed mutagenesis using the QuikChange Lightning Multi Site-Directed Mutagenesis Kit (Agilent Technologies, part no. 210514). The mutagenized trimers 426C.DS.SOSIP, WITO 501GCG SOSIP.664 (containing a modified furin cleavage site), BG505 375Y SOSIP.664 and JR-FL 375Y SOSIP.664 (containing a tyrosine substitution at position 375 ) were expressed by co-transfecting the mutagenized plasmids with a plasmid expressing the cellular protease furin into Free-style 293-F cells (ThermoFisher Scientific). For AD8, a non-SOS soluble trimer was used that was devoid of the stabilizing 501-605 disulfide bond but which contained a neo-disulfide bond between amino acids 113 and 432 (ref. ${ }^{17}$ ). This trimer design will be described elsewhere (Zhang et al., in preparation). Cell-free supernatants were collected after 6-7 days by centrifugation and passed through a $0.22 \mu \mathrm{m}$ filter. A Galanthus nivalis lectin affinity column (Vector Laboratories) and size-exclusion column were used to purify the trimer proteins. The Env trimer protein corresponding to the peak was collected and concentrated, and then processed in a monoclonal antibody 447-52D affinity column for negative selection of open trimer forms. Purified trimers were concentrated to $0.5-2 \mathrm{mg} \mathrm{ml}^{-1}$ in PBS and stored at $-80^{\circ} \mathrm{C}$

In vitro mRNA transfection and flow cytometry assays. HEK293T cells (obtained from ATCC) were transiently transfected with mRNA encoding different HIV-1 Envs using the TransIT-mRNA Transfection Kit (Mirus). After $48 \mathrm{~h}$ the cells were collected by mechanical shaking and pipetting, washed with PBS, and incubated with CD4-Ig or anti-gp120 antibodies $\left(1 \mu \mathrm{g} \mathrm{ml}^{-1}\right)$ in flow cytometry buffer ( $1 \times$ $\mathrm{PBS}$ with $2 \% \mathrm{FBS}$ ) at room temperature for $30 \mathrm{~min}$. After washing with PBS twice, the cells were incubated with phycoerythrin (PE)-conjugated goat anti-human IgG (Southern Biotech) at room temperature for $15 \mathrm{~min}$. The cells were then washed once with PBS, fixed in $2 \%$ paraformaldehyde (PFA) and analyzed on a BD LSRFortessa (BD Biosciences). Data analysis and geometric mean fluorescence intensity measurement were performed using FlowJo v10.7.1.

Antibody-binding assays. HIV-1 Env binding by antibodies present in macaque plasma or serum was tested using ELISA as previously described ${ }^{17}$. For assessing endpoint binding titers, 96-well ELISA plates (Corning) were either coated with $4 \mu \mathrm{g} \mathrm{ml}^{-1}$ Galanthus nivalis lectin (Sigma) for testing Env trimer binding or directly coated with smaller antigens (for example, SIV p27 Gag, V3 peptides, gp70-V1V2 scaffold, or eOD-GT8 and related proteins) at $1 \mu \mathrm{g} \mathrm{ml}^{-1}$ in PBS at $4^{\circ} \mathrm{C}$ overnight. Then, the plates were blocked with $1 \times$ casein at room temperature for $1 \mathrm{~h}$. All the antibodies and HIV-1 Env proteins used in ELISA experiments were diluted in $0.1 \times$ casein solution in PBS (washing buffer). All samples were washed three times with washing buffer after each step. To evaluate macaque sera binding to soluble Env trimers, purified SOSIP trimers $\left(1 \mu \mathrm{g} \mathrm{ml}^{-1}\right)$ were added to lectin-coated plates for $1 \mathrm{~h}$ at room temperature. After washing, serially diluted rhesus macaque sera were added and incubated for $1 \mathrm{~h}$ at room temperature. After washing, HRP-conjugated goat anti-human IgG (Jackson ImmunoResearch), cross-reactive with macaque IgG, was added for $1 \mathrm{~h}$ at room temperature. The reaction was revealed by incubation with $50 \mu \mathrm{l}$ substrate (R\&D Systems) for $15 \mathrm{~min}$ before the 
addition of $25 \mu$ stop solution. Light absorption was measured at a wavelength of $450 \mathrm{~nm}$. All samples were tested in duplicate wells.

bNAb competition ELISA. BG505 SOSIP.664 trimers $\left(1 \mu \mathrm{g} \mathrm{ml}{ }^{-1}\right)$ were added to lectin-coated ELISA plates for $1 \mathrm{~h}$ at RT. Macaque sera (at 1:100 dilution) and biotinylated bNAbs or sCD4-4d at pre-determined concentrations to yield $50-60 \%$ of maximum binding signal were added to the plates simultaneously. Bound bNAbs were detected using Streptavidin-HRP (R\&D Systems). The optical density at $450 \mathrm{~nm}$ was measured and the binding data were analyzed with Prism 8 (GraphPad Prism Software). The degree of competition was calculated as the percentage of signal reduction in the presence or absence of the immune macaque sera.

HIV-1 pseudovirus production and neutralization assays. Plasmids expressing various HIV-1 Envs were co-transfected with an HIV-1 backbone plasmid (pSG3 $\Delta$ env, Env-deficient) into HEK293T cells. One $\mu \mathrm{g}$ of each Env-expressing plasmid and $1 \mu \mathrm{g}$ of the backbone plasmid were mixed in Opti-MEM medium (Gibco), and $4 \mu \mathrm{l} \mathrm{LiFect} 293$ Transfection Reagent were added at a 1:2 DNA: reagent ratio. The DNA-LiFect293 Transfection Reagent complex was incubated for $15 \mathrm{~min}$ at room temperature and added to $10^{6} \mathrm{HEK} 293 \mathrm{~T}$ cells in 6-well plates with $2 \mathrm{ml}$ fresh $10 \%$ FBS-DMEM. After incubation for 2 days at $37^{\circ} \mathrm{C}$, the supernatants containing pseudoviruses were centrifuged, filtered by a $0.45 \mu \mathrm{m}$ filter, aliquoted and titrated for further use. For single-round HIV-1 Env-pseudovirus infection of TZM-bl cells, heat-inactivated rhesus macaque sera were serially diluted threefold and incubated with the pseudoviruses for $30 \mathrm{~min}$ at room temperature; then, $10^{4} \mathrm{TZM}$-bl cells were added to 96 -well flat-bottom plates in $10 \%$ FBS-DMEM, in a total volume of $200 \mu \mathrm{l}$ per well. All the samples were tested in duplicate or triplicate wells. After $72 \mathrm{~h}$ the medium was completely removed and the reporter gene (luciferase) expression was detected by adding $50 \mu \mathrm{l}$ per well Luciferase Assay Reagent (Promega). The cell lysates were transferred to luminescence plates and measured with a luminometer (PerkinElmer). The viral stocks were used at a concentration yielding approximately 100,000-500,000 relative light units (RLU). $\mathrm{IC}_{50}$ values were calculated using a dose-response curve fit with a 5-parameter non-linear function using GraphPad Prism 8

Macaque CD4 functional assay. Rhesus macaque CD4 (rhCD4) and HIV-1 co-receptor-expressing HEK293T cells were generated by transient transfection of rhCD4, rhCXCR4 and rhCCR5 expression vectors (NIH HIV Reagent Program, cat. nos. 3600, 3602 and 3601) on the day before the pseudovirus titration experiment. Titrated HIV-1 WT or 375Y pseudoviruses were incubated with the transfected HEK293T cells. Seventy-two hours later, the cells were lysed and luciferase activity (RLU) was measured.

Cell-surface staining and ADCC assay. For cell-surface staining, SHIV AD8-infected primary human CD4 ${ }^{+}$T cells were stained with AquaVivid viability dye and incubated with heat-inactivated macaque sera (at 1:100 dilution) for $1 \mathrm{~h}$ at $37^{\circ} \mathrm{C}$. Cells were washed three times with cold PBS before adding Alexa Fluor 647-conjugated anti-human IgG secondary antibody (Invitrogen). Productively infected cells were identified by intracellular staining for SIV p27 (antibody clone 2F12) and CD4 (antibody clone OKT4) as $27^{+} \mathrm{CD} 4^{\text {low }}$ cells. For ADCC, infected cells were stained with AquaVivid viability dye and cell proliferation dye (eFluor670, eBioscience) and used as target cells. Autologous PBMC effector cells, stained with proliferation dye eFluor450 (eBioscience), were added at an effector: target ratio of 10:1 in 96-well V-bottom plates (Corning). Then, heat-inactivated macaque sera (at 1:100 dilution) were added to appropriate wells and the cells were incubated for $15 \mathrm{~min}$ at room temperature. Subsequently, the cells were centrifuged for $1 \mathrm{~min}$ at $300 \mathrm{x} g$ and incubated at $37^{\circ} \mathrm{C}$ in $5 \% \mathrm{CO}_{2}$ for $5 \mathrm{~h}$ before being fixed in a $2 \%$ PFA solution in PBS. Samples were acquired on an LSRII cytometer (BD Biosciences) and data analysis was performed using FlowJo vX.0.7 (Tree Star). The percentage of cytotoxicity was calculated using the following formula: $\left(\%\right.$ of $\mathrm{p} 27^{+} \mathrm{CD} 4^{\text {low }}$ cells in targets plus effectors $)-\left(\%\right.$ of $\mathrm{p} 27^{+} \mathrm{CD} 4^{\text {low }}$ cells in targets plus effectors plus sera $) /(\%$ of $\mathrm{p} 27^{+} \mathrm{CD} 4^{\text {low }}$ cells in targets) by gating on infected live target cells.

T cell immunity assays. Cryopreserved PBMC were thawed into warm R10 media (RPMI 1640, 10\% FBS, $2 \mathrm{mM}$ L-glutamine, $100 \mathrm{U} \mathrm{ml}^{-1}$ penicillin and $100 \mu \mathrm{g} \mathrm{ml}^{-1}$ streptomycin), washed and rested for $1 \mathrm{~h}$ before stimulation. For stimulation, $1-1.5$ million cells were plated into 96-well V-bottom plates in $200 \mu \mathrm{l} \mathrm{R} 10$ and stimulated with SIV Gag or HIV-1 clade-B Env peptides $\left(2 \mu \mathrm{g} \mathrm{ml}^{-1}\right.$; NIH AIDS Reagent Program) in the presence of Brefeldin A $\left(10 \mu \mathrm{g} \mathrm{ml}^{-1}\right.$; Sigma-Aldrich) and monensin (GolgiStop; BD Biosciences). Titrated amounts of anti-CD107a and anti-CXCR3 were included in the culture during cell stimulation. A DMSO-only condition was included to determine background for unstimulated cells. Following stimulation for $6 \mathrm{~h}$, samples were stained with LIVE/DEAD Fixable Blue Dead Cell Stain for $10 \mathrm{~min}$ at room temperature and surface stained with titrated amounts of anti-CD14, anti-CD20, anti-CD4, anti-CXCR5, anti-CD28, anti-PD-1, anti-CD8, anti-CD95 and anti-CCR7 for $20 \mathrm{~min}$ at room temperature. Cells were washed in FACS Buffer (PBS + 2\% FBS), fixed, and permeabilized (Cytofix/Cytoperm; BD Biosciences) for $20 \mathrm{~min}$ at room temperature. Cells were washed with Perm/Wash buffer (BD Biosciences) and stained intracellularly with anti-CD3, anti-CD154, anti-interferon- $\gamma$ (anti-IFN- $\gamma$ ), anti-IL-2, anti-IL-4, anti-IL-21, anti-tumor necrosis factor- $\alpha$ (anti-TNF- $\alpha$ ), anti-Granzyme B and anti-CD69 for $20 \mathrm{~min}$ at room temperature. The antibodies used for staining are listed as follows. The directly conjugated antibodies purchased from BD Biosciences were CD107a BV510 (Clone H4A3; cat. no. 563078), CXCR3 PE-Cy5 (Clone 1C6/CXCR3; cat. no. 551128), CD14 BUV805 (Clone M5E2; cat. no. 612902), CD20 BUV805 (Clone 2H7; cat. no. 612905), CD4 BUV395 (Clone L200; cat. no. 564107), CD28 BB515 (Clone CD28.2; cat. no. 564492), CD8 BUV496 (Clone RPA-T8; cat. no. 612942), CD3 APC-Cy7 (Clone SP34-2; cat. no. 557757), IFN- $\gamma$ V450 (Clone B27; cat. no. 560371), IL-2 BUV737 (Clone MQ1-17H12; cat. no. 612836) and IL-21 Alexa Fluor 647 (Clone 3A3N2.1; cat. no. 560493). The antibodies purchased from Biolegend were PD-1 BV785 (Clone EH12.2H7; cat. no. 329930), CD95 BV605 (Clone DX2; cat. no. 305628), CCR7 BV650 (Clone G043H7; cat. no. 353234); CD154 BV711 (Clone 24-31; cat. no. 310838), TNF- $\alpha$ Alexa Fluor 700 (Clone Mab11; cat. no. 502928) and CD69 PE (Clone FN50; cat. no. 310906). CXCR5 PE-eFluor 610 (Clone 5UMUBEE; cat. no. 61-9185-42), IL-4 PE-Cy7 (Clone 8D4-8; cat. 25-704982) and Granzyme B PE-Cy5.5 (Clone GB11; cat. no. GRB18) were purchased from Invitrogen along with LIVE/DEAD Fixable Blue Dead Cell Stain (cat. no. L34962). After intracellular staining the cells were washed with Perm/Wash buffer, fixed with $1 \%$ PFA, and data were acquired on a modified BD FACSymphony. Data were analyzed using FlowJo v10.7.1, and the cytokine frequencies were background subtracted. Examples of the gating strategy are shown in Supplementary Fig. 1. Polyfunctional T cell responses were analyzed using SPICE, as reported ${ }^{82}$.

Plasma viral RNA quantification assay. Plasma SHIV RNA levels were measured using a gag-targeted quantitative real-time PCR with reverse transcription (RT-PCR) assay. Plasma RNA was extracted using a QIAamp Viral RNA Kit (Qiagen) and reverse-transcribed with the AgPath-ID One-Step RT-PCR Kit (Applied Biosystems). The primers, probe and amplification conditions were as previously reported ${ }^{83}$. The samples were measured in triplicate reactions, and the copy numbers were calculated by interpolation of a standard curve generated with a digested SIV gag DNA fragment that contains the RT-PCR amplicon (serial 10 -fold dilutions from 1 copy per reaction to $10^{6}$ copies per reaction).

Polyclonal epitope mapping by NSEM. Fabs were generated from macaque sera using the Pierce Fab Preparation Kit following the manufacturer's protocol. In brief, macaque IgG was purified by affinity columns of Protein A-Sepharose resin (GE Healthcare). After washing with PBS (10-fold the column volume), IgG was eluted by IgG-elution buffer (Thermo Fisher) and immediately neutralized with pH 9.0 Tris-HCl buffer. Resin-immobilized papain $(250 \mu \mathrm{l}$ settled resin for $4 \mathrm{mg}$ IgG, Thermo Fisher Scientific) was prepared and equilibrated in the digestion

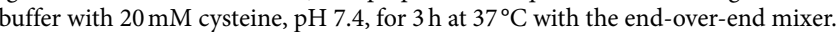
Heavy chain constant fragments $(\mathrm{Fc})$ and undigested IgG were removed by passage over Protein A-Sepharose. The Fab-containing supernatant was buffer exchanged into PBS by centrifugation with an Amico MWCO 10,000 Da centrifuge filter unit (EMD Millipore). Polyclonal Fabs were diluted to a concentration 10-fold higher than that of BG505 SOSIP.664 trimer, and the mixtures of Fab and BG505 were incubated at $4^{\circ} \mathrm{C}$ overnight in PBS. The complexes were diluted to a concentration of $\sim 0.02 \mathrm{mg} \mathrm{ml}^{-1}$, adsorbed to freshly glow-discharged carbon-coated grids for $15 \mathrm{~s}$ washed with a buffer containing $10 \mathrm{mM}$ HEPES, $\mathrm{pH}$ 7, and $150 \mathrm{mM} \mathrm{NaCl}$, and stained with $0.7 \%$ uranyl formate. Images were collected on a Thermo Scientific Talos F200C electron microscope operating at $200 \mathrm{kV}$ and equipped with a $4 \mathrm{k} \times 4 \mathrm{k}$ Ceta CCD camera at a nominal magnification of 57,000 , corresponding to a pixel size of $0.253 \mathrm{~nm}$. Particles were picked from micrographs automatically using an in-house written software (Y.T., unpublished data). Reference-free two-dimensional classification was performed in Relion ${ }^{84}$

Statistical analysis. The protective efficacy of the vaccine was evaluated based on the time to infection over the course of 13 sequential weekly low-dose inoculations. The Kaplan-Meier curves of vaccinated and control macaques were provided and compared using the Wilcoxon exact test. Vaccine efficacy, defined as 1 minus the hazard ratio, was estimated from a Cox proportional hazards regression using exact partial likelihood with group (vaccinated versus control) as the regressor. The association of various immunological parameters with vaccine protection was assessed using Spearman's rank correlation with the number of infection-free days post-challenge.

Reporting Summary. Further information on research design is available in the Nature Research Reporting Summary linked to this article.

\section{Data availability}

The GenBank accession codes for the mRNA sequences are MZ362872, MZ362873, MZ362874, MZ362875 and MZ362876.

\section{References}

79. Nelson, J. et al. Impact of mRNA chemistry and manufacturing process on innate immune activation. Sci. Adv. 6, eaaz6893 (2020)

80. Hassett, K. J. et al. Optimization of lipid nanoparticles for intramuscular administration of mRNA vaccines. Mol. Ther. Nucleic Acids 15, 1-11 (2019). 
81. Bahl, K. et al. Preclinical and clinical demonstration of immunogenicity by mRNA vaccines against $\mathrm{H} 10 \mathrm{~N} 8$ and $\mathrm{H} 7 \mathrm{~N} 9$ influenza viruses. Mol. Ther. 25, 1316-1327 (2017).

82. Roederer, M., Nozzi, J. L. \& Nason, M. C. SPICE: exploration and analysis of post-cytometric complex multivariate datasets. Cytometry A 79, 167-174 (2011).

83. Vassena, L. et al. Treatment with IL-7 prevents the decline of circulating CD4+ T cells during the acute phase of SIV infection in rhesus macaques. PLoS Pathog. 8, e1002636 (2012).

84. Scheres, S. H. RELION: implementation of a Bayesian approach to cryo-EM structure determination. J. Struct. Biol. 180, 519-530 (2012).

\section{Acknowledgements}

The authors thank the animal care personnel at Bioqual Inc. and at the Poolesville NIH Animal Facility. The authors also thank J. Moore (Weill-Cornell Medicine, New York) for the BG505 SOSIP.664 plasmid, J. Van Schooten (University of Amsterdam, The Netherlands) for plasmids to produce monoclonal antibodies RM20A3, RM20C, RM20E1, RM20F, RM20G, RM20J, RM15E and RM54B1, and the National Institutes of Health (NIH) AIDS Reagent Program for the reagents listed in the Methods section. This study was supported by the Intramural Research Program of the Division of Intramural Research and the Vaccine Research Center, National Institute of Allergy and Infectious Diseases, NIH, by a grant from the NIH Office of AIDS Research, by the Bill and Melinda Gates Foundation, and by federal funds from the Frederick National Laboratory for Cancer Research, NIH, under contract HHSN261200800001 (to Y.T.)

\section{Author contributions}

P.Z. and P.L. conceived and supervised the project; P.Z., E.N., S.D., K.B., Y.L.,

Q.L., H.M., H.S., D.R., S.F., S.M.E., V.P., K.B., M.P., X.C., E.K.S. and D.R.A. performed the experiments; D.W., J.M., J.S. and R.H. provided veterinary care and performed the immunizations and viral challenges; R.G. and M.A.M. provided critical reagents and expertise for the NHP challenge study; Y.T. performed the NSEM analysis; Z.H. and D.F. performed the statistical analysis; A.F., G.C., S.H., G.S.-J., A.C., A.M.D., R.A.K., J.R.M. and A.S.F. participated in the study design; P.Z. and P.L. wrote the original draft of the paper; all of the authors contributed to the final review of the paper and the editing.

\section{Competing interests}

P.L., P.Z., and E.N. are named on a HIV RNA Vaccine patent (PCT/US2020/ 022710). E.N., S.F., S.M.E., V.P., K.B., S.H., G.S.-J. and A.C. are employees of Moderna Inc. and hold equities from the company. All other authors have no competing interests.

\section{Additional information}

Extended data are available for this paper at https://doi.org/10.1038/ s41591-021-01574-5.

Supplementary information The online version contains supplementary material available at https://doi.org/10.1038/s41591-021-01574-5.

Correspondence and requests for materials should be addressed to Paolo Lusso. Peer review information Nature Medicine thanks Joseph Jardine and the other, anonymous, reviewer(s) for their contribution to the peer review of this work. Alison Farrell was the primary editor on this article and managed its editorial process and peer review in collaboration with the rest of the editorial team.

Reprints and permissions information is available at www.nature.com/reprints. 

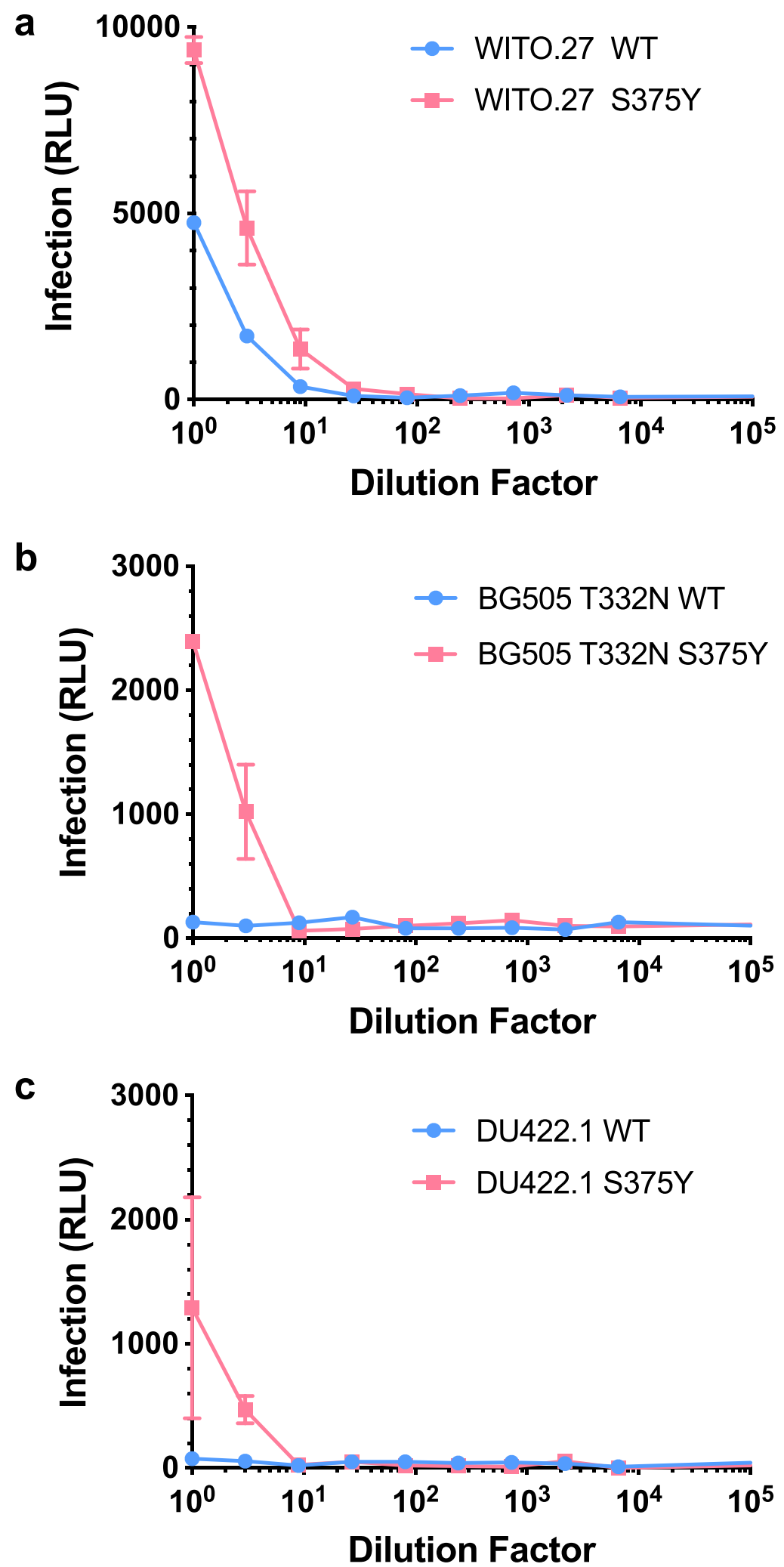

Extended Data Fig. 1 | Utilization of macaque CD4 by wild-type and mutated HIV-1 Envs. Ability of three wild-type (WT) and corresponding aa. 375-mutated HIV-1 strains to utilize rhesus macaque CD4 (rhCD4) for infection. rhCD4 transfection and pseudovirus infection are described in the Methods section. 
a

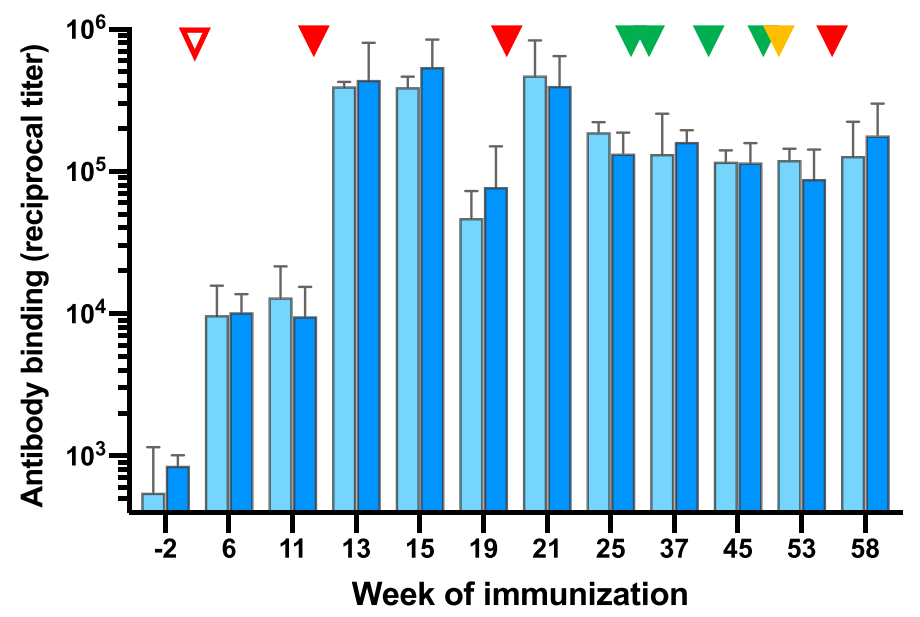

C

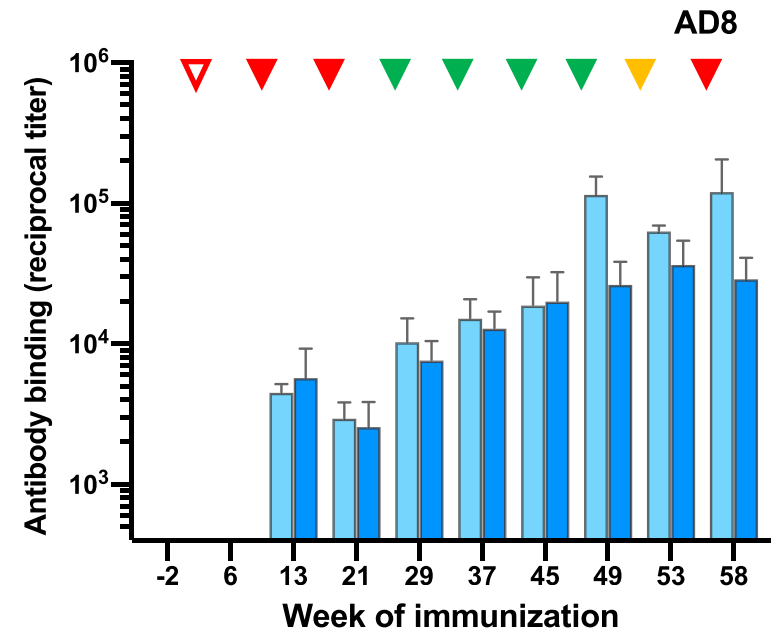

b

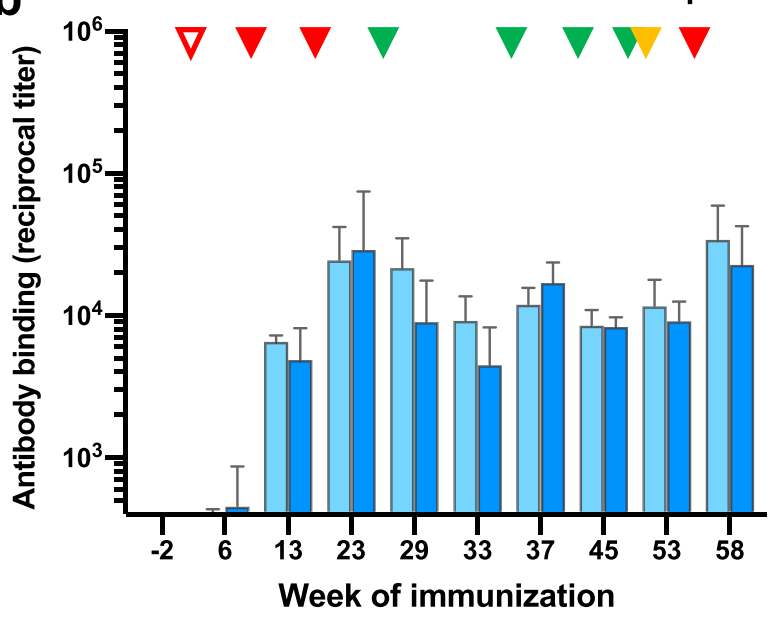

Vaccine subgroups:

Subgroup 1

Subgroup 2

Immunizations:

$\nabla$ Autologous Env open (WITO $\Delta 276$ )

$\nabla$ Autologous Env closed (WITO)

$\checkmark$ Heterologous Envs (BG505+DU422)

$\nabla$ Heterologous Env (JR-FL)

Extended Data Fig. 2 | Induction of trimer-binding antibodies in the two subgroups of immunized macaques over time. Subgroup 1 received 5 mRNA and 5 protein immunizations; subgroup 2 received 8 mRNA and 2 protein immunizations. a. Serum titers of antibodies binding to the autologous trimer as assayed by ELISA using the WITO SOSIP.664 trimer captured on lectin-coated plates. b. Serum titers of antibodies binding to the autologous V3 loop peptide (WITO pV3) as assayed using the linear WITO V3 peptide directly coated on the plate surface. c. Serum titers of antibodies binding to a heterologous trimer (AD8) as assayed by ELISA using the AD8 IP trimer captured on lectin-coated plates. Statistical differences were calculated using a paired two-tailed t-test. The sequence and timing of immunizations are indicated by color-coded triangles on the top. 
a

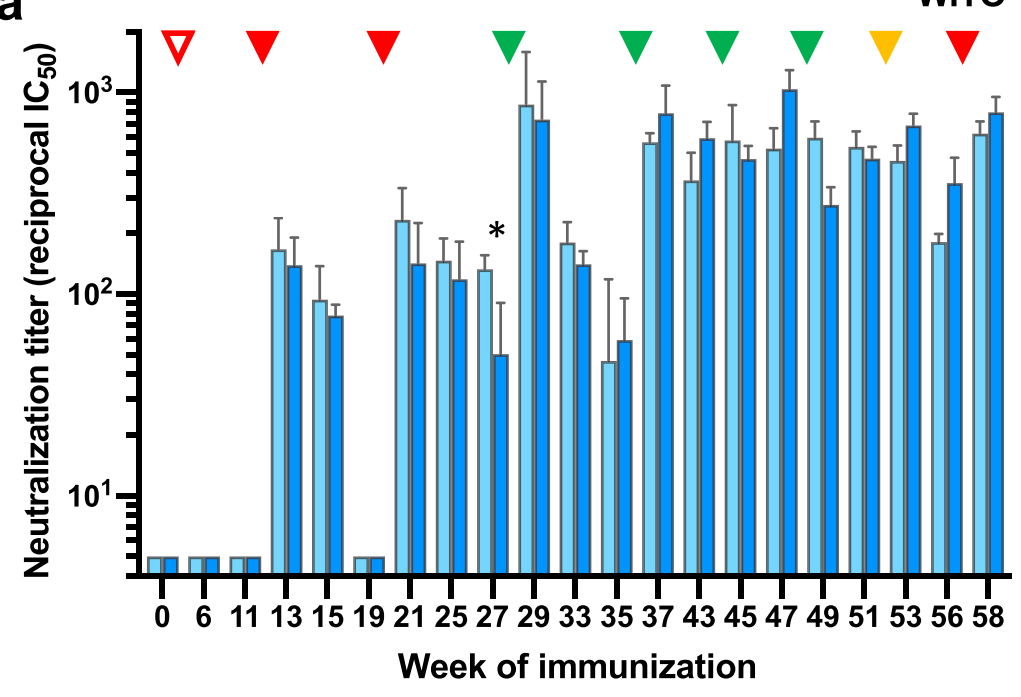

b

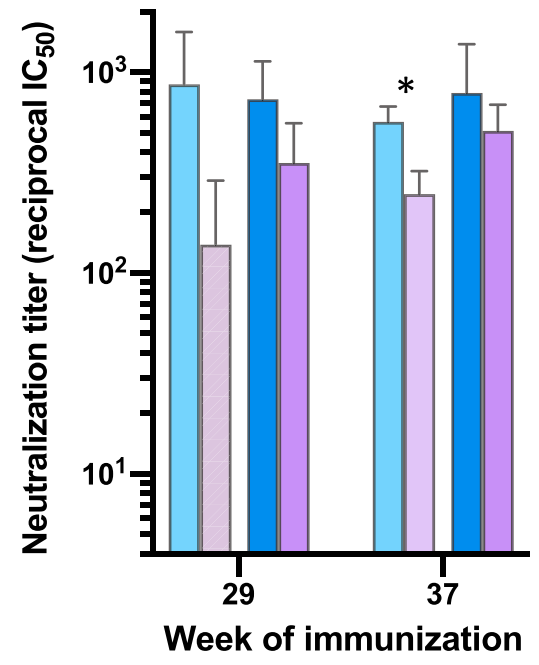

\section{Immunizations:}

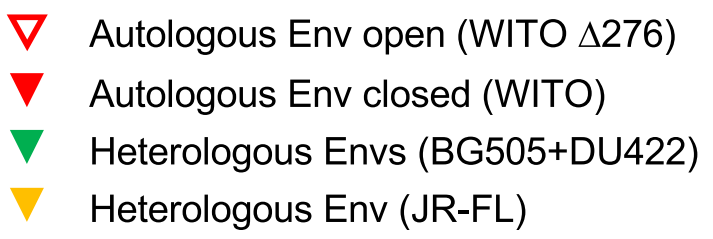

\section{Vaccine subgroups:}

$\begin{array}{ll}\text { Subgroup } 1 & \square+\mathrm{V} 3 \text { peptide } \\ \text { Subgroup } 2 & \square+\mathrm{V} 3 \text { peptide }\end{array}$
$+\mathrm{V} 3$ peptide

Extended Data Fig. 3 | Induction of autologous neutralizing antibodies in the two subgroups of immunized macaques. Subgroup 1 received 5 mRNA and 5 protein immunizations; subgroup 2 received 8 mRNA and 2 protein immunizations. a. Serum titers of neutralizing antibodies against the autologous Env (WITO4160.27), as assayed using pseudoviruses on TZM-bl cells. The sequence and timing of immunizations are indicated by color-coded triangles on the top. b. Effect of the autologous linear V3-loop peptide (WITO pV3) on autologous neutralization at two time points (week 29 and 37 ). The assays were performed by incubating the linear $\mathrm{V} 3$ peptide with macaque sera prior to addition to the pseudovirus stock. The asterisk denotes a $p$ value $<0.05$, as assessed by two-tailed paired t-test. 
a

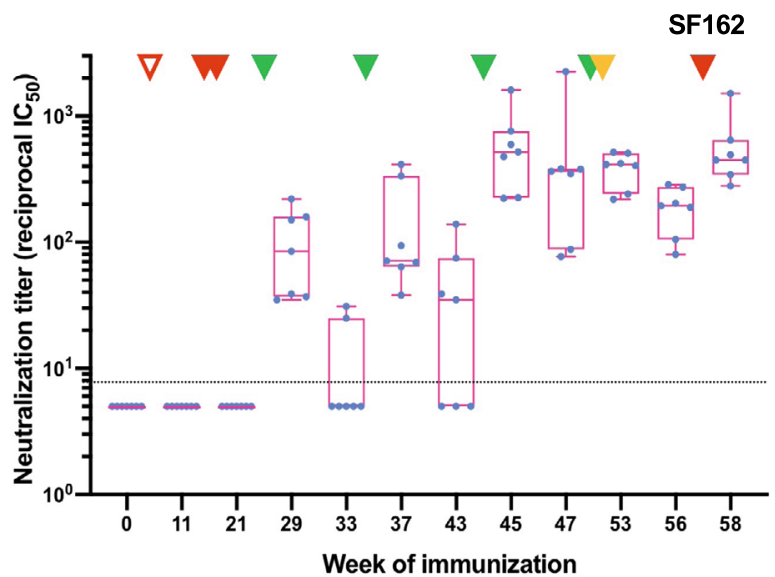

C

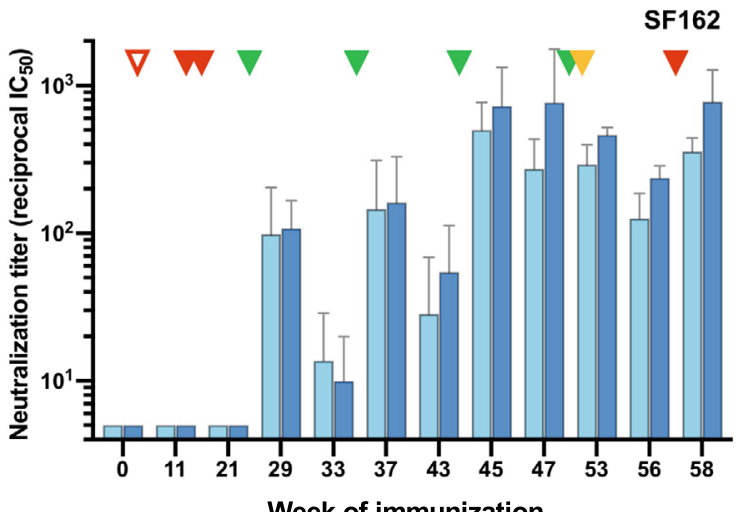

e

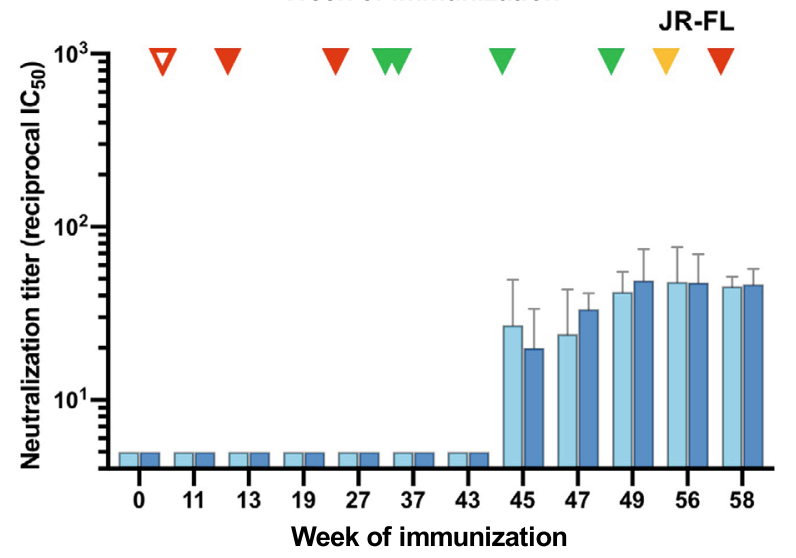

g

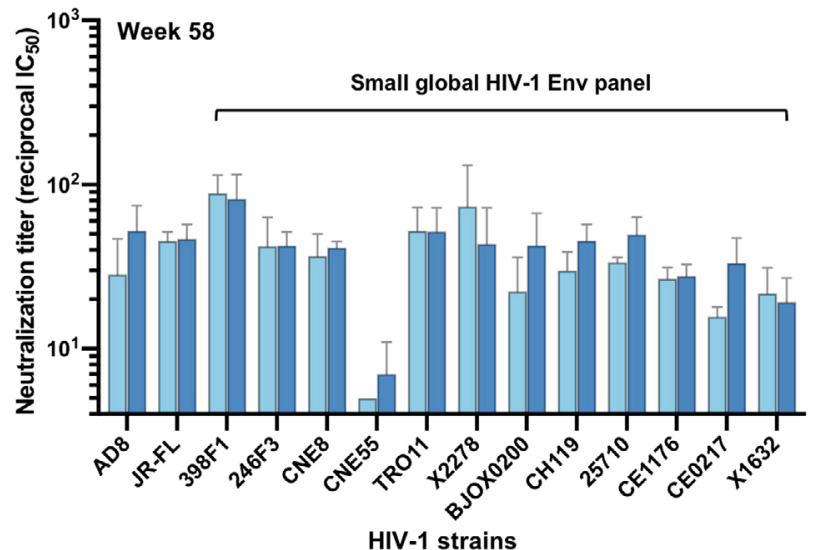

b

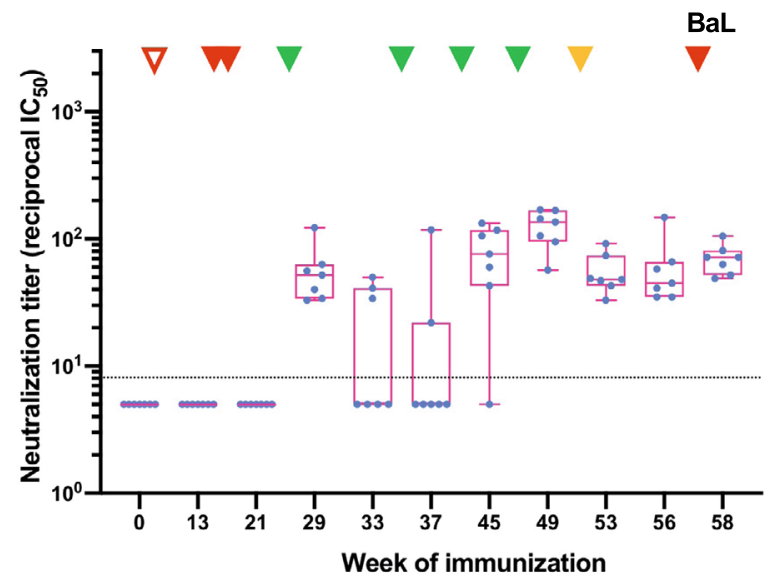

d

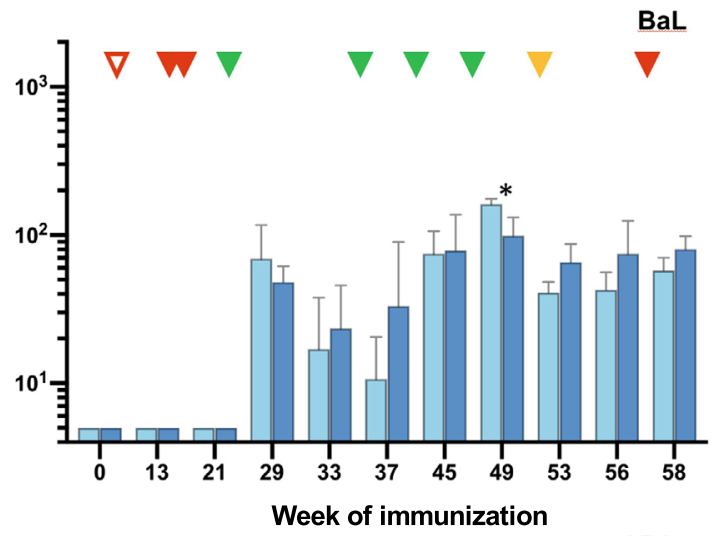

f

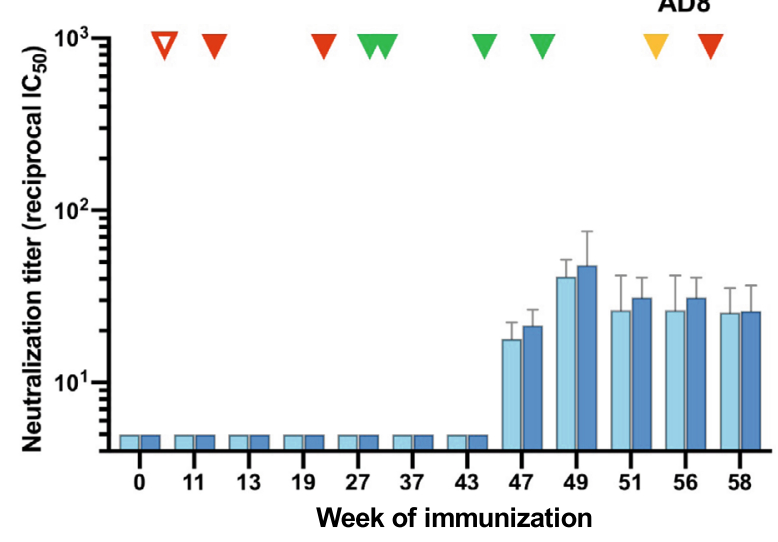

Extended Data Fig. 4 | See next page for caption.

Vaccine subgroups:

Subgroup 1

Subgroup 2

Immunizations:

$\nabla$ Autologous Env open (WITO $\Delta 276$ )

$\checkmark$ Autologous Env closed (WITO)

$\nabla$ Heterologous Envs (BG505+DU422)

$\checkmark$ Heterologous Env (JR-FL) 
Extended Data Fig. 4 | Induction of heterologous neutralizing antibodies in immunized macaques. a. Serum titers of neutralizing antibodies against a heterologous tier-1a Env (SF162). b. Serum titers of neutralizing antibodies against a heterologous tier-1b Env (BaL). c. Neutralization of SF162 in the two vaccine subgroups. $\mathbf{d}$. Neutralization of BaL in the two vaccine subgroups. The asterisk denotes a $\mathrm{p}$ value $<0.05$, as assessed by two-tailed unpaired t-test. e. Neutralization of the tier-2 Env JR-FL in the two vaccine subgroups. f. Neutralization of the tier-2 Env AD8 in the two vaccine subgroups. g. Serum titers of neutralizing antibodies against a panel of heterologous tier-2 Envs at week 58 in the two vaccine subgroups. Neutralization assays were performed using pseudoviruses on TZM-bl cells. The sequence and timing of immunizations are indicated by color-coded triangles on the top. Subgroup 1 received 5 mRNA and 5 protein immunizations; subgroup 2 received 8 mRNA and 2 protein immunizations. 
a

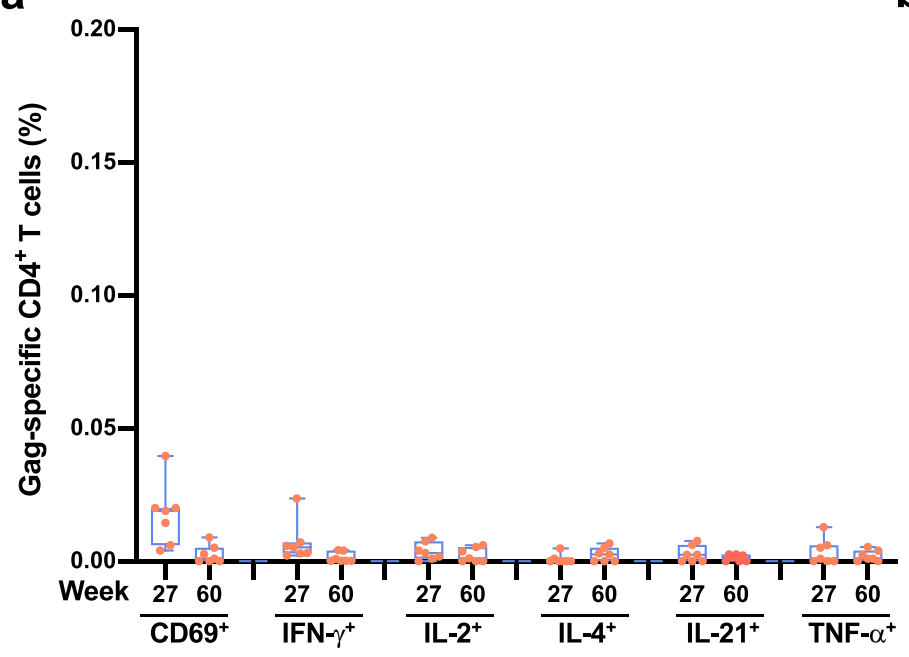

b

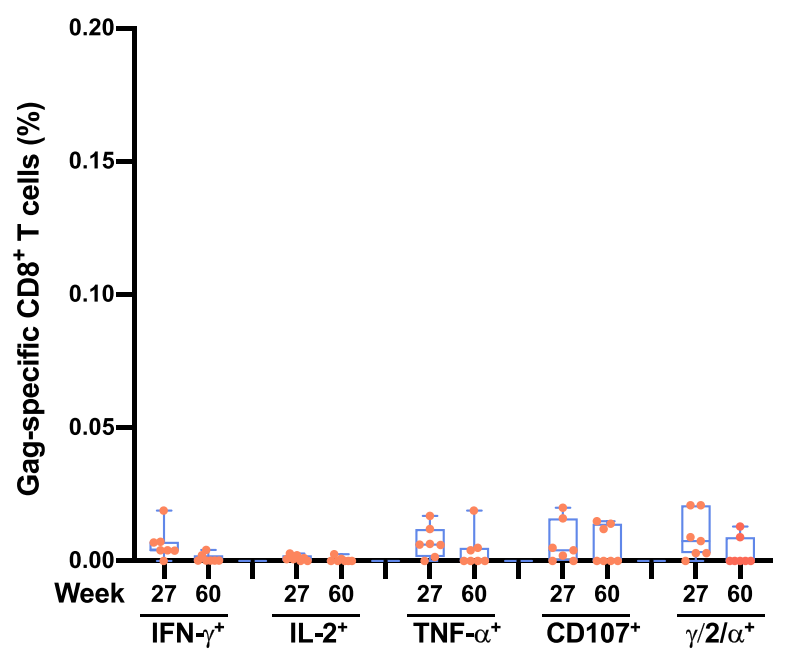

C

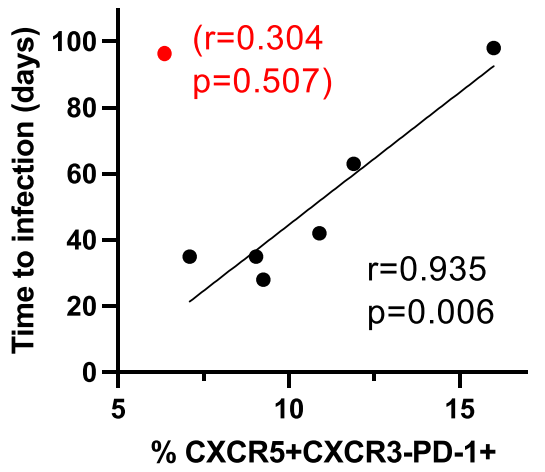

Extended Data Fig. 5 | SIV Gag-specific T-cell responses. Induction of SIV Gag-specific CD4 ${ }^{+}$and CD8 ${ }^{+}$T-cell responses in all 7 vaccinated macaques at weeks 27 and 60 of immunization. a. Gag-specific CD4+ $4^{+}$-cell responses calculated as fractions of memory CD4 ${ }^{+}$T cells expressing CD154 and the indicated intracellular cytokines or CD69. b. Gag-specific CD8+ T-cell responses calculated as fractions of memory CD8 ${ }^{+} \mathrm{T}$ cells expressing CD69 and the indicated intracellular cytokines or CD107. c. Correlation between a subset of Env-specific T-follicular helper cells (CXCR5 ${ }^{+}$CXCR3-PD-1 ${ }^{+}$) and protection in vaccinated macaques. The correlation was statistically significant after exclusion of a single outlier (macaque $\mathrm{V} 7$, highlighted in red). The correlation coefficient and $\mathrm{P}$ value with the inclusion of the outlier are indicated in red. 


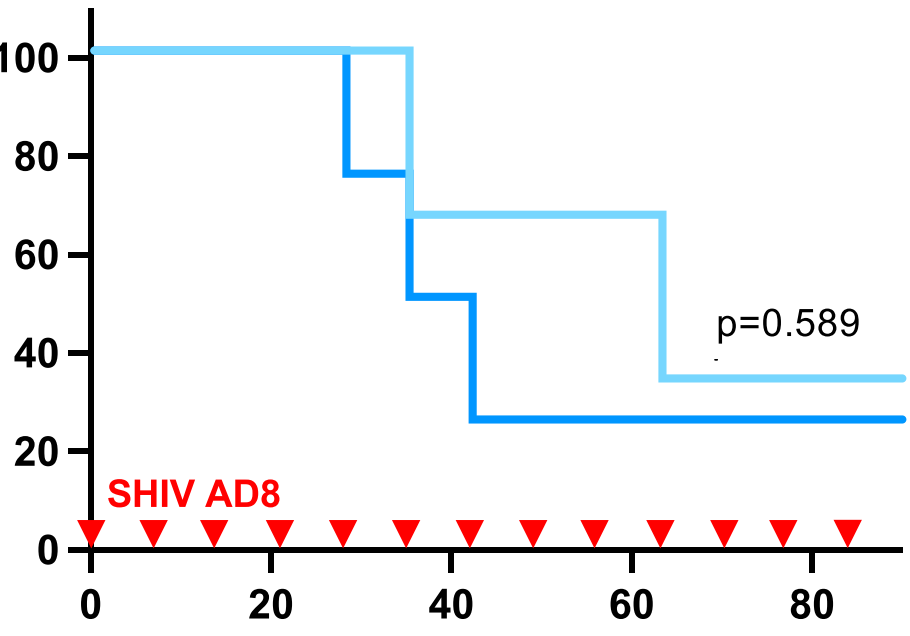

Time to infection (days)

b

\begin{tabular}{|c|c|c|c|c|c|c|}
\hline & \multirow{2}{*}{$\begin{array}{c}\text { Hazard } \\
\text { ratio }\end{array}$} & \multicolumn{2}{|c|}{$95 \% \mathrm{Cl}$} & \multirow{2}{*}{$\begin{array}{l}\text { Per-exposure } \\
\text { risk reduction }\end{array}$} & \multicolumn{2}{|c|}{$95 \% \mathrm{Cl}$} \\
\hline & & lower & upper & & lower & upper \\
\hline Subgroup 1 & 0.14 & 0.02 & 1.28 & $86 \%$ & $1 \%$ & $98 \%$ \\
\hline Subgroup 2 & 0.31 & 0.06 & 1.62 & $76 \%$ & $1 \%$ & $94 \%$ \\
\hline
\end{tabular}

C

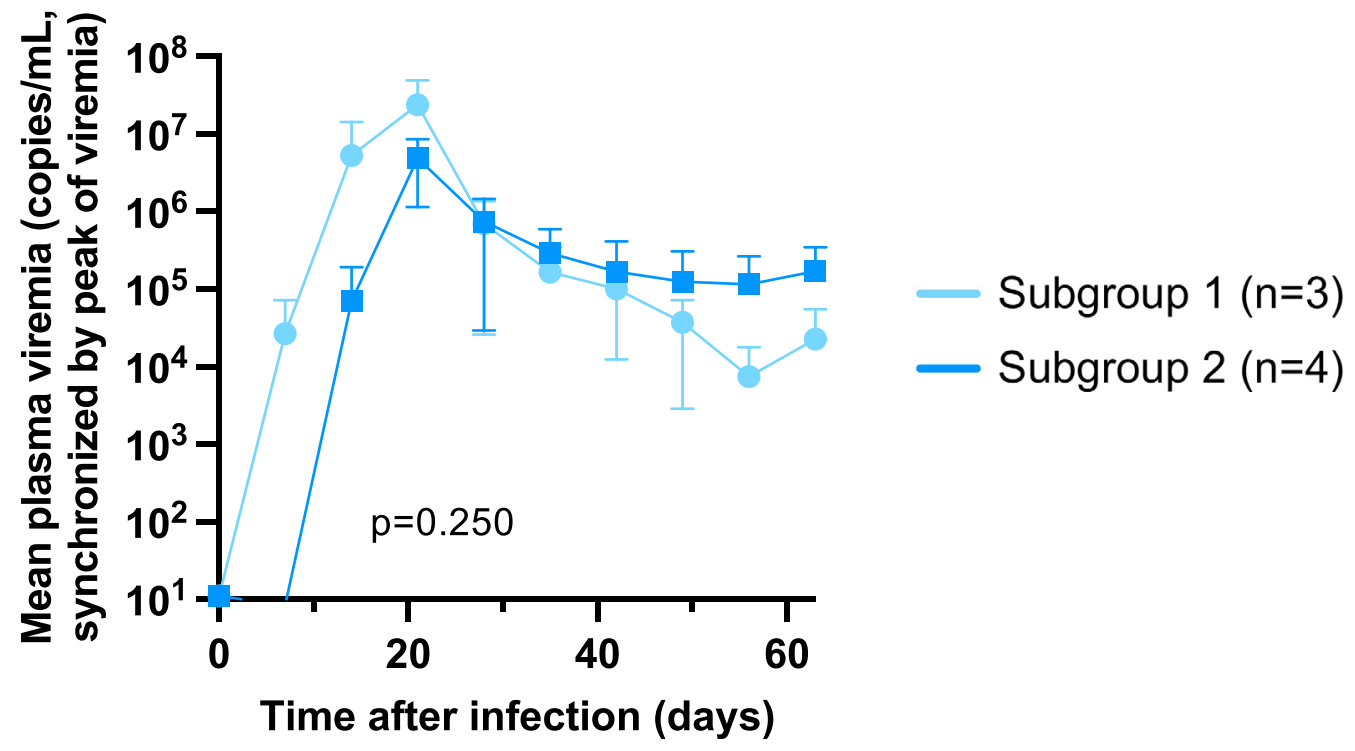

Extended Data Fig. 6 | Vaccine-induced protection in the two subgroups of immunized macaques challenged with SHIV AD8. Subgroup 1 received 5 mRNA and 5 protein immunizations; subgroup 2 received 8 mRNA and 2 protein immunizations. a. Kaplan-Meier analysis of virus-free survival in the course of 13 weekly intrarectal inoculation of 10 TCID $_{50}$ of SHIV AD8 (red arrows) in the two subgroups of immunized macaques. Infection was evaluated by the appearance of plasma viremia on two subsequent tests using a sensitive real-time PCR method. Significance was calculated by the Wilcoxon exact test. b. Statistical analysis of hazard ratio and per-exposure risk in the two subgroups of immunized macaques, as assessed by 1 minus the hazard ratio, estimated from a Cox proportional hazards regression via the exact partial likelihood with group (vaccinated versus control) as the regressor. c. Mean levels of viremia $( \pm S D)$ in macaques in the two vaccine subgroups synchronized by peak of viremia. Levels of viremia were evaluated by quantitative real-time PCR. The courses of viremia in different animals were aligned by setting the peak of viremia at day 21 for each animal. 
a
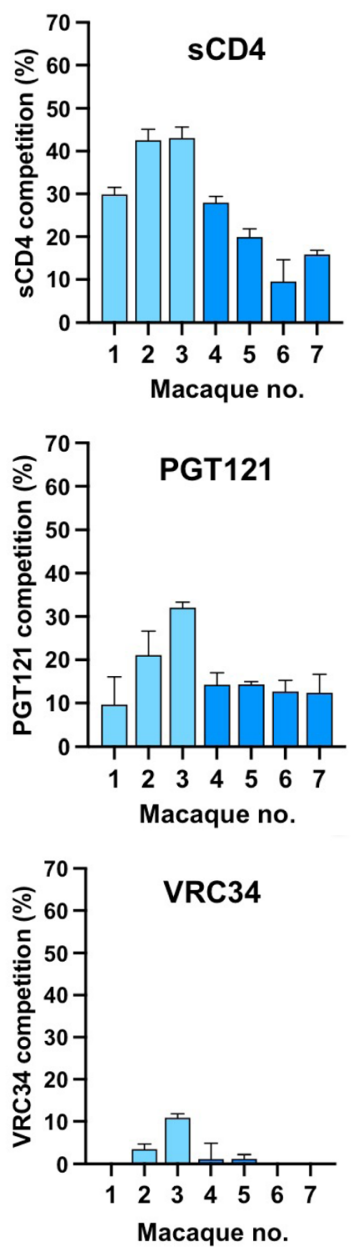

b
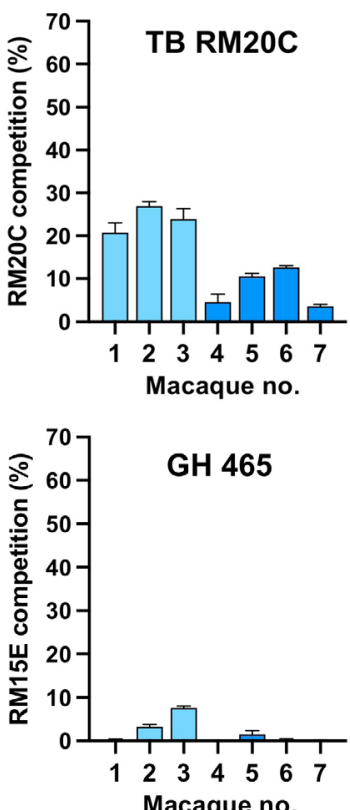
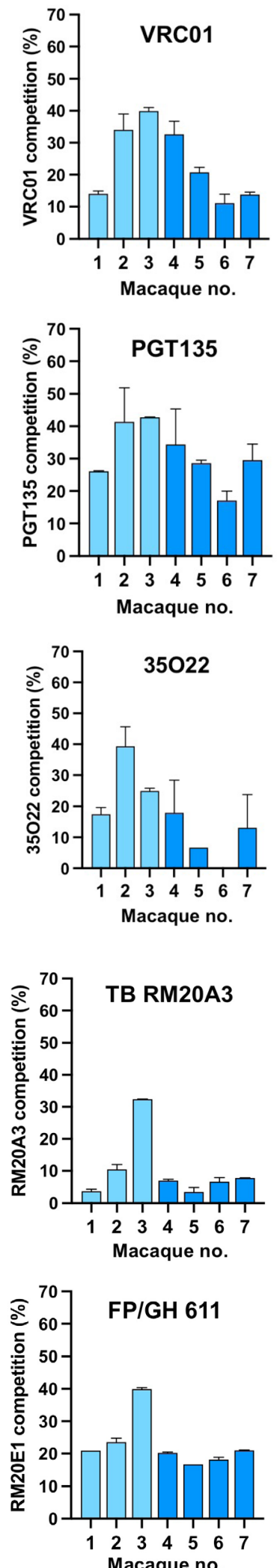
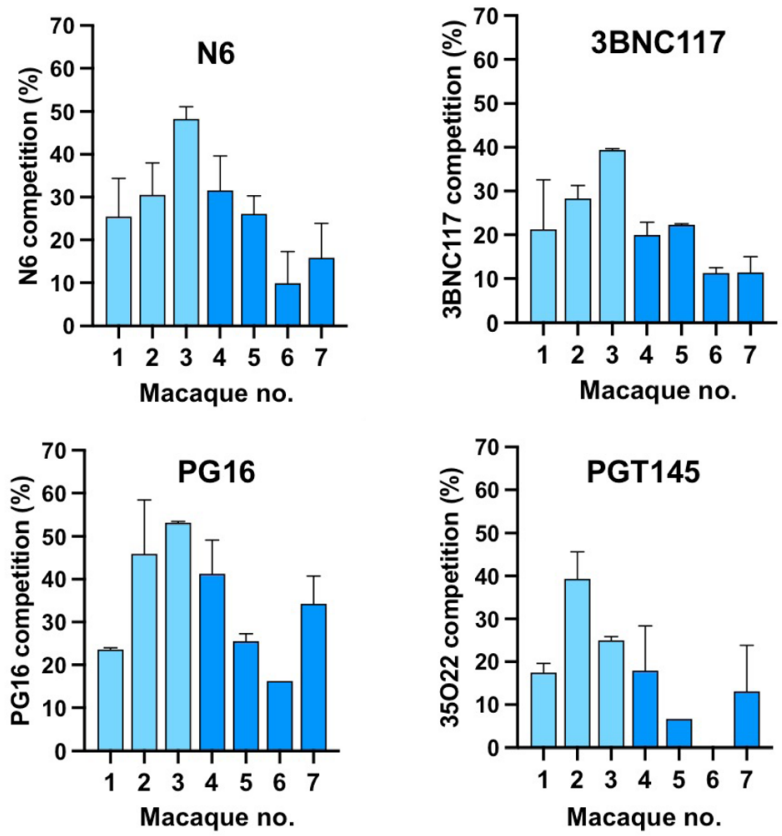

Vaccine subgroups:

Subgroup 1

Subgroup 2
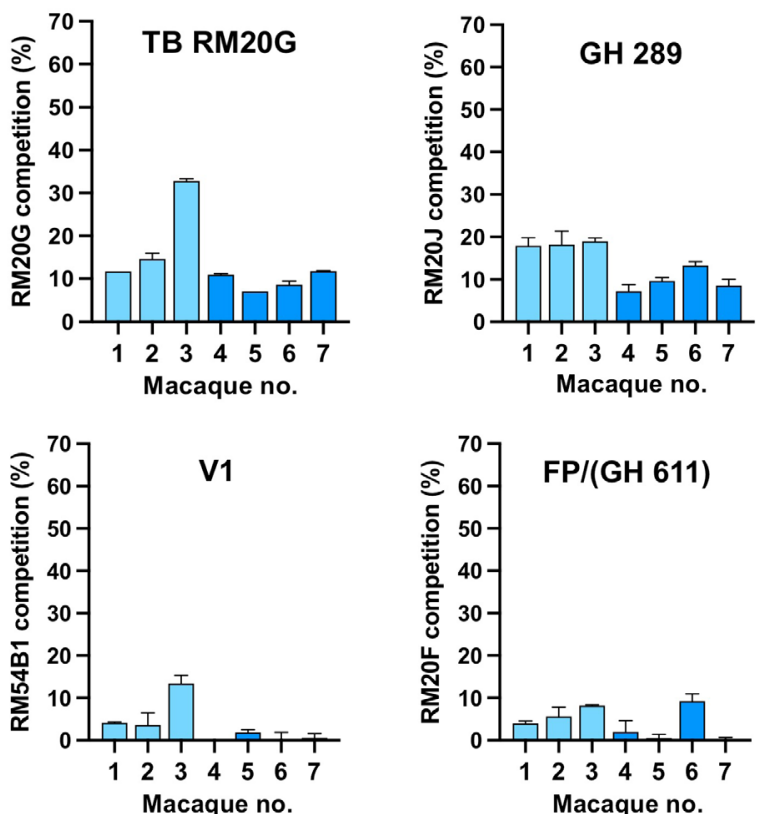

Extended Data Fig. 7 | Epitope mapping of trimer-binding antibodies in serum from vaccinated macaques. a. Competition with HIV-1 bNAbs, as assessed by ELISA on lectin-captured HIV-1 BG505 SOSIP.664 trimers. b. Competition with macaque monoclonal antibodies directed against off-target and non-bNAb-target regions (RM20A3, RM20C, RM20E1, RM2OF, RM20G, RM20J, RM15E and RM54B1). TB: trimer base; GH: glycan holes; V1: V1 loop; FP: fusion peptide. 
a
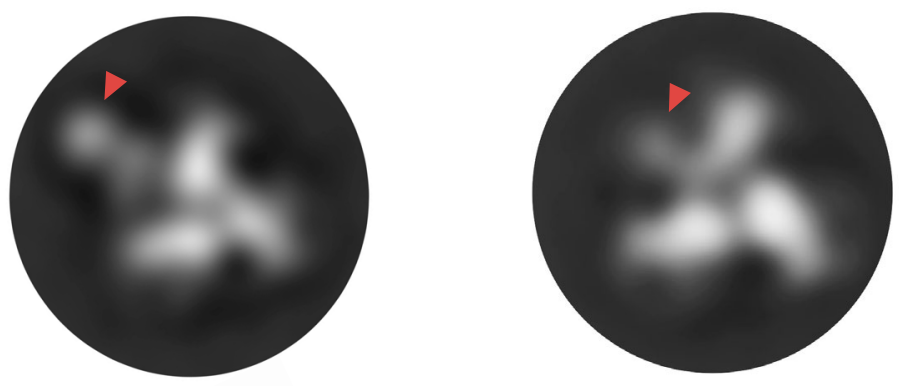

b
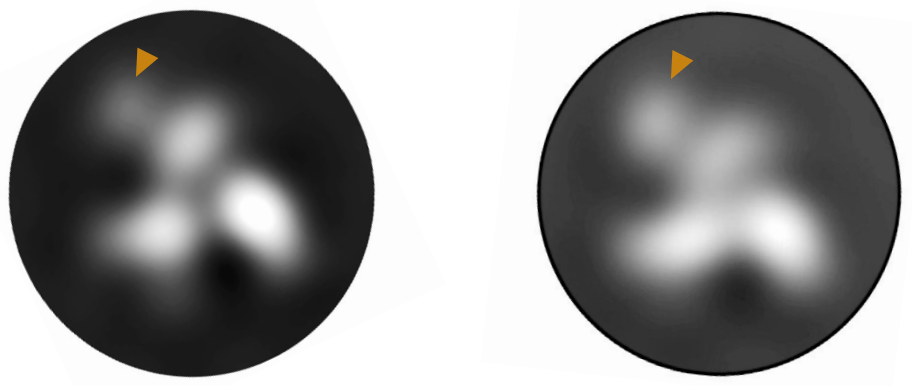

C
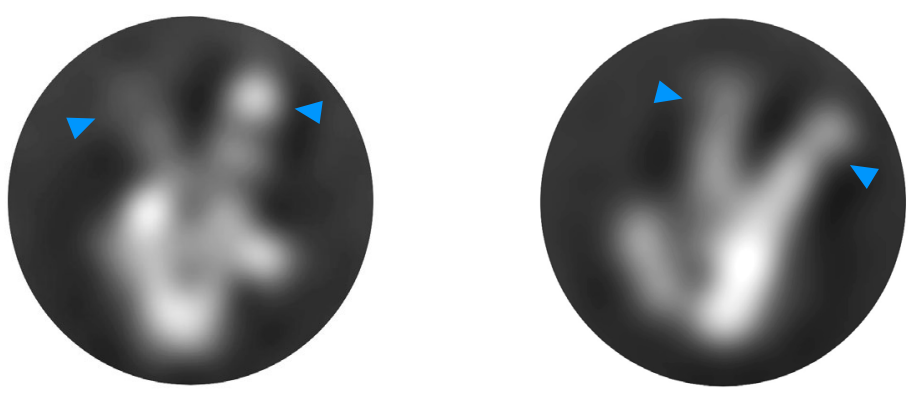

d
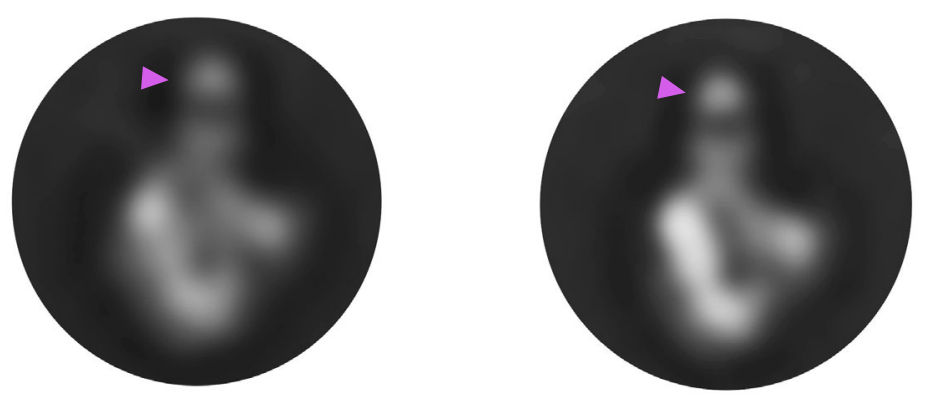

e
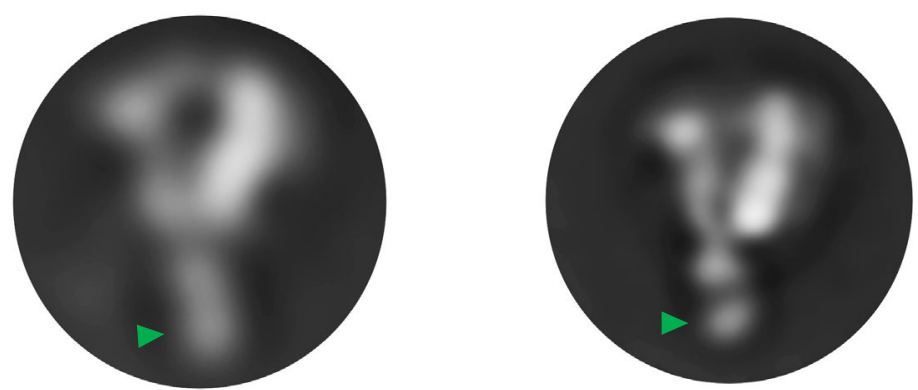

Extended Data Fig. 8 | Polyclonal epitope mapping of trimer-binding antibodies in a representative protected animal by negative-staining electron microscopy (NSEM). The analysis was performed using purified Fab fragments isolated from serum collected from macaque V3 (subgroup 1) at week 58. The arrow heads point to the trimer-bound Fab fragments. Visualization of antibodies binding to: $\mathbf{a}$. A region compatible with the CD4-BS deep in the inter-protomer groove; b. A region in the inter-protomer groove more distal from the trimer axis than the CD4-BS; $\mathbf{c}$. A region(s) at the trimer apex targeted by 2 Fabs simultaneously; d. A region at the trimer apex compatible with the V2-glycan supersite targeted by a single Fab; e. A region at the trimer base, presumably constituted by neo-epitopes produced by gp 41 truncation. 
a

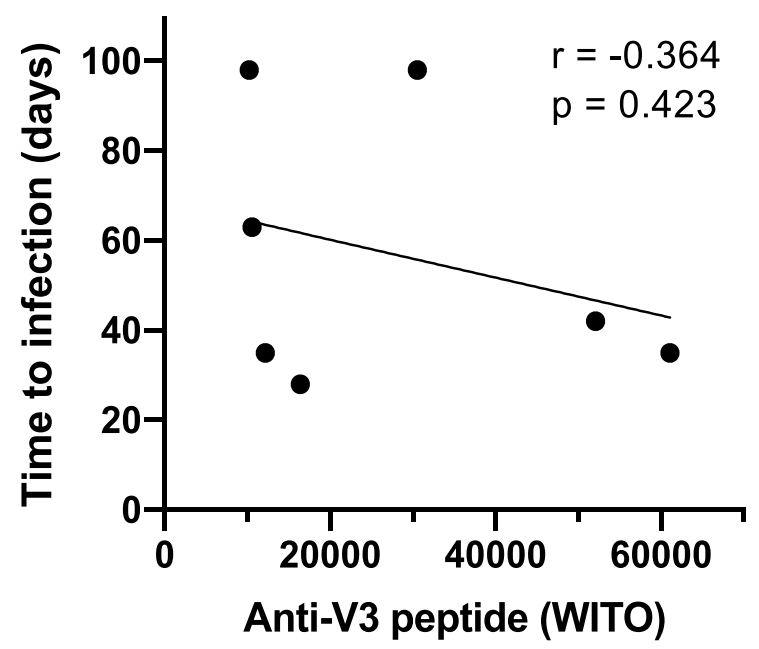

C

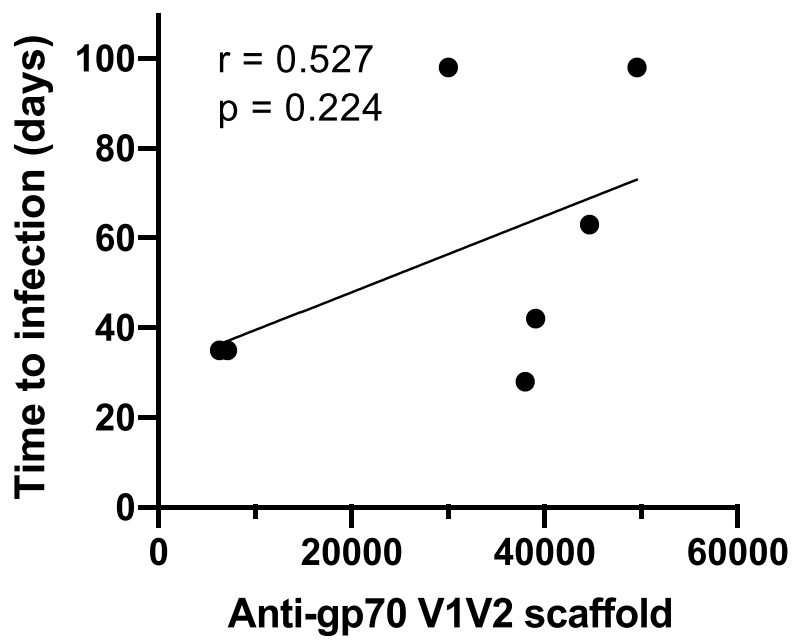

e

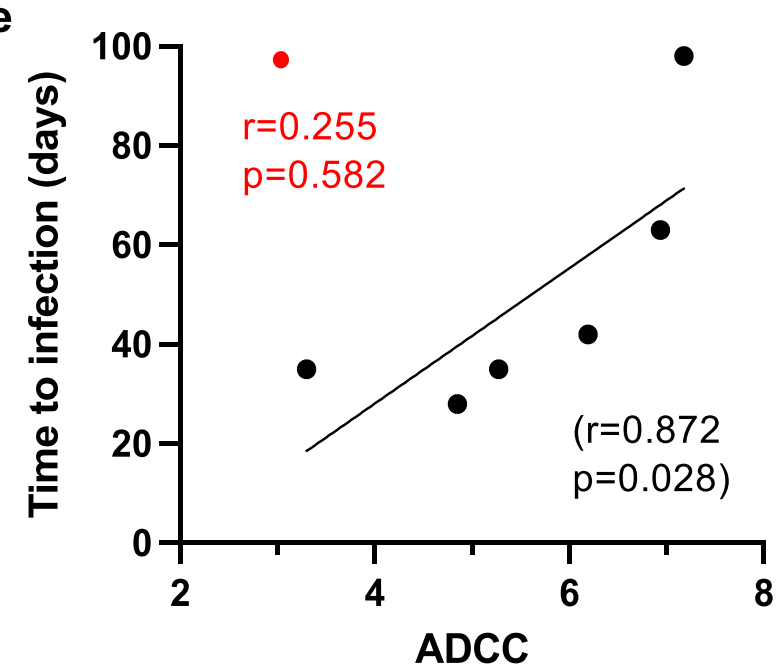

b

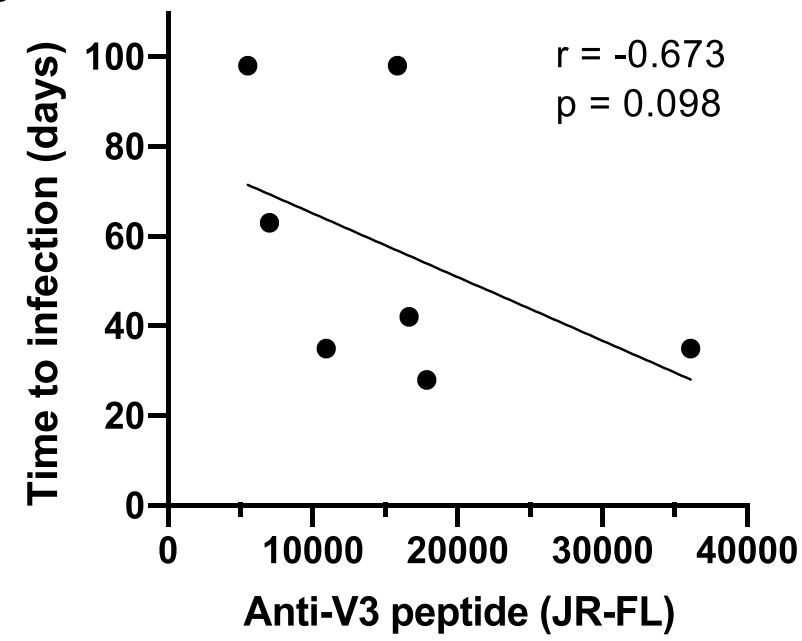

d

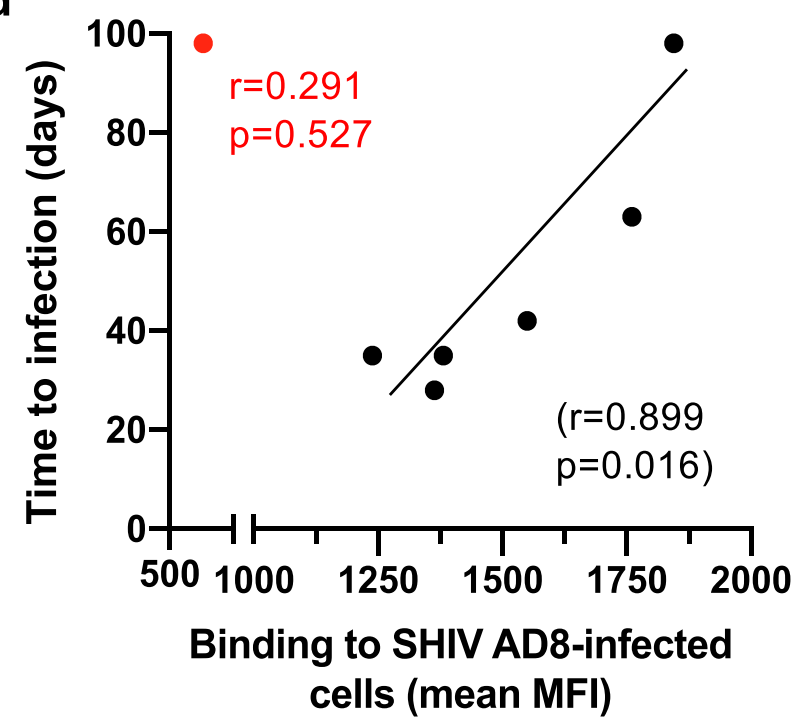

f

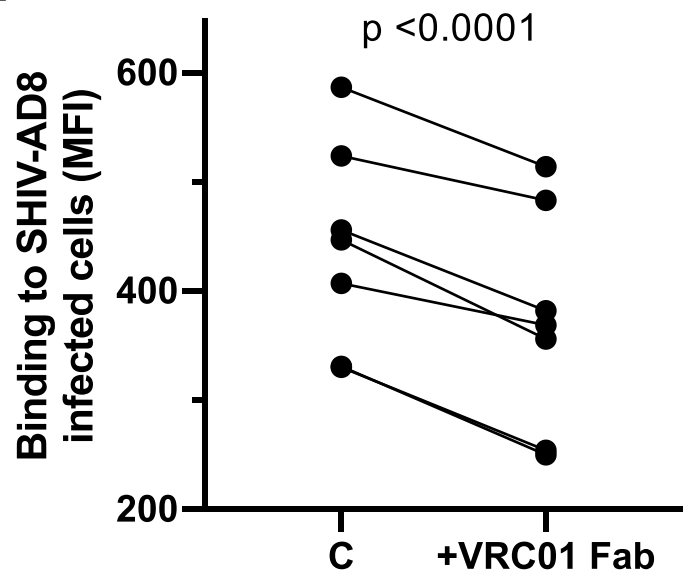


Extended Data Fig. 9 | Correlation between vaccine-induced protection and antibodies against specific epitopes, infected cells or ADCC. a. Correlation between protection and antibodies against an autologous linear V3 peptide (WITO pV3). b. Correlation between protection and antibodies against a heterologous linear V3 peptide (JR-FL V3). c. Correlation between protection and antibodies against a V1V2 scaffolded protein (gp70 V1V2). d. Correlation between protection and antibodies binding to the surface of SHIV AD8-infected primary CD4 ${ }^{+} \mathrm{T}$ cells. The results are mean values from two experiments. The correlation becomes significant after exclusion of an outlier (macaque $\mathrm{V} 7$, highlighted in red). The correlation coefficient and $\mathrm{P}$ value with the inclusion of the outlier are indicated in red. e. Correlation between protection and antibodies mediating ADCC against SHIV AD8-infected primary CD4+ T cells. The results are mean values from two experiments. The correlation becomes significant after exclusion of an outlier (macaque V1, highlighted in red). The correlation coefficient and $P$ value with the inclusion of the outlier are indicated in red. $\mathbf{f}$. Inhibitory effect of a VRC01 Fab fragment on macaque antibody binding to SHIV AD8-infected primary CD4+ $\mathrm{T}$ cells. $\mathrm{C}$ denotes Fab-untreated controls. All correlations were determined by the Spearman test. 


\section{Reporting Summary}

Nature Research wishes to improve the reproducibility of the work that we publish. This form provides structure for consistency and transparency in reporting. For further information on Nature Research policies, see our Editorial Policies and the Editorial Policy Checklist.

\section{Statistics}

For all statistical analyses, confirm that the following items are present in the figure legend, table legend, main text, or Methods section.

$\mathrm{n} / \mathrm{a}$ Confirmed

\ The exact sample size $(n)$ for each experimental group/condition, given as a discrete number and unit of measurement

$\bigotimes$ A statement on whether measurements were taken from distinct samples or whether the same sample was measured repeatedly

The statistical test(s) used AND whether they are one- or two-sided

Only common tests should be described solely by name; describe more complex techniques in the Methods section.

$\bigotimes$ A description of all covariates tested

Х $\square$ A description of any assumptions or corrections, such as tests of normality and adjustment for multiple comparisons

$\square$ A full description of the statistical parameters including central tendency (e.g. means) or other basic estimates (e.g. regression coefficient)

AND variation (e.g. standard deviation) or associated estimates of uncertainty (e.g. confidence intervals)

For null hypothesis testing, the test statistic (e.g. $F, t, r$ ) with confidence intervals, effect sizes, degrees of freedom and $P$ value noted Give $P$ values as exact values whenever suitable.

Х $\square$ For Bayesian analysis, information on the choice of priors and Markov chain Monte Carlo settings

Х $\square$ For hierarchical and complex designs, identification of the appropriate level for tests and full reporting of outcomes

$\square \bigotimes$ Estimates of effect sizes (e.g. Cohen's $d$, Pearson's $r$ ), indicating how they were calculated

Our web collection on statistics for biologists contains articles on many of the points above.

\section{Software and code}

Policy information about availability of computer code

Data collection

BD FACSDiva Software version 9.0 for FACS

Enspire Manager version for 413.3005.1482 for Elisa and Neutralization

EPU version 2.2.0.65REL for NSEM data

Data analysis FlowJo 10.7.1 for FACS analysis; Microsoft Excel 16.36;

GraphPad Prism 8.4.2;

Microsoft Excel 16.49;

SPICE 6 version6.1(61001)

MacVector version 17.0.5 (43)

For statistical analysis, R software, version 4.0.3.

Relion version 1.4 for NSEM data

For manuscripts utilizing custom algorithms or software that are central to the research but not yet described in published literature, software must be made available to editors and reviewers. We strongly encourage code deposition in a community repository (e.g. GitHub). See the Nature Research guidelines for submitting code \& software for further information. 
Policy information about availability of data

All manuscripts must include a data availability statement. This statement should provide the following information, where applicable:

- Accession codes, unique identifiers, or web links for publicly available datasets

- A list of figures that have associated raw data

- A description of any restrictions on data availability

All mRNA sequence data are publicly available (Accession codes: MZ362872, MZ362873, MZ362874, MZ362875, MZ362876).

\section{Field-specific reporting}

Please select the one below that is the best fit for your research. If you are not sure, read the appropriate sections before making your selection.

Х Life sciences

Behavioural \& social sciences

Ecological, evolutionary \& environmental sciences

For a reference copy of the document with all sections, see nature.com/documents/nr-reporting-summary-flat.pdf

\section{Life sciences study design}

All studies must disclose on these points even when the disclosure is negative.

Sample size

For the mouse study a Wilcoxon sum rank test with two-tailed significance level of 0.05 would have over $80 \%$ power to detect an effective size of 1.6 between the Env and Env-Gag vaccine arms, each with the sample size of 8, and an effect size of 2 between a vaccine arm and the control arm with the sample size of 8 and 4 , respectively. For the macaque study, with seven rhesus macaques per arm, the study had over $80 \%$ power to detect the protective efficacy of the vaccine against HIV-1 infection if the protective efficacy, defined as one minus the hazard ratio, was no less than 0.90 from a Wilcoxon sum rank test on the time to infection with two-tailed significance level of 0.05 .

Data exclusions No data were excluded from this report

Replication All critical assays were reliably replicated as indicated in figures or tables.

Animal experiments were carried out in experimental groups. All immunized animals developed both trimer-binding and neutralizing antibodies.

Randomization Mice were inbred from the same colony and the same age range. They were randomly assigned to the study groups. Macaques were randomly assigned to the study groups, which were balanced for age, weight, CBC (complete blood count) and chemistry profiles.

Blinding $\quad$ Mice and macaques were identified by ear tags or skin tatoos, respectively, and therefore could not be handled blindly. However, operators were blinded to the substances administered to each group.

\section{Reporting for specific materials, systems and methods}

We require information from authors about some types of materials, experimental systems and methods used in many studies. Here, indicate whether each material, system or method listed is relevant to your study. If you are not sure if a list item applies to your research, read the appropriate section before selecting a response.

Materials \& experimental systems

$\mathrm{n} / \mathrm{a}$ Involved in the study

$\square$ Antibodies

$\square$ Eukaryotic cell lines

\ $\square$ Palaeontology and archaeology

$\square \bigotimes$ Animals and other organisms

$\bigotimes \square$ Human research participants

Х $\square$ Clinical data

\ $\square$ Dual use research of concern

\begin{tabular}{l} 
Methods \\
\hline n/a Involved in the study \\
$\square$ \\
$\square$ ChIP-seq \\
$\square$ Flow cytometry \\
$\square$ MRI-based neuroimaging
\end{tabular}

\section{Antibodies}

Antibodies used
Previously published human monoclonal antibodies were obtained from the AIDS Research Program, including CD4-Ig VRC01 VRC03 VRC07 VRC-PG04 VRC-CH31 N6 N49P7 3BNC117 NIH45-46 HJ16 F105 b6 b12 b13 10-1074 PGT121 PGT123 PGT125 PGT126 PGT128 PGT130 PGT135 PGT136 2G12 PG9 PG16 PGT145 CH01 CH02 CH03 VRC26.08 VRC38 447-52D 19B 39F b4e8 2219 3074 412D 48d 17b 35022 8ANC195 PGT151 VRC34 2 F5 4E10 10E8. VRC01-UCA CH31-UCA CH103-UCA CH01-UCA PG9-UCA RM20J RM2OF RM20E1 RM20G RM20C RM20A3 RM54B1 RM15E were produced in our laboratories based on published sequences. The antigenic profile of these antibodies, assessed by ELISA and flow cytometry, agrees with published descriptions as referenced throughout the 
Validation

manuscript. Directly conjugated antibodies purchased from BD Biosciences include CD107a BV510 (Clone H4A3; cat. 563078), CXCR3 PE-Cy5 (Clone 1C6/CXCR3; cat. 551128), CD14 BUV805 (Clone M5E2; cat. 612902), CD20 BUV805 (Clone 2H7; cat. 612905), CD4 BUV395 (Clone L200; cat 564107), CD28 BB515 (Clone CD28.2; cat. 564492), CD8 BUV496 (Clone RPA-T8; cat. 612942), CD3 APC-Cy7 (Clone SP34-2; cat. 557757), IFN-gamma V450 (Clone B27; cat. 560371), IL-2 BUV737 (Clone MQ1-17H12; cat. 612836) and IL-21 Alexa Fluor 647 (Clone 3A3N2.1; cat. 560493). Antibodies purchased from Biolegend include PD-1 BV785 (Clone EH12.2H7; cat. 329930), CD95 BV605 (Clone DX2; cat. 305628), CCR7 BV650 (Clone G043H7; cat. 353234); CD154 BV711 (Clone 24-31; cat. 310838), TNF-alpha Alexa Fluor 700 (Clone Mab11; cat. 502928) and CD69 PE (Clone FN50; cat. 310906). CXCR5 PE-eFluor 610 (Clone 5UMUBEE; cat. 61-9185-42), IL-4 PE-Cy7 (Clone 8D4-8; cat. 25-7049-82) and Granzyme B PE-Cy5.5 (Clone GB11; cat. GRB18) were purchased from Invitrogen along with LIVE/DEAD Fixable Blue Dead Cell Stain (cat. L34962).

The primary antibodies program were extensively validated on appropriate positive and negative controls in our experiments. The information about antibodies from HIV-1 reagent could be found at https://www.hivreagentprogram.org/Home.aspx. The catalog information is also listed here: ARP-857 Antibody (F105), ARP-1475 Antibody (2F5), ARP-1476 Antibody (2G12), ARP-1756 Antibody (48d),ARP-4030 Antibody (447-52D),ARP-4091 Antibody 17b, ARP-10091 Antibody (4E10),ARP-11437 Antibody (39F), ARP-11438 Antibody (A32),ARP-11682 gp120 Antibody (2191), ARP-12032 Antibody (VRC03),ARP-12033 Antibody (VRC01), ARP-12040 Antibody (3074), ARP-12138 Antibody (HJ16), ARP-12149 Antibody (PG9), ARP-12150 Antibody (PG16),ARP-12294 Antibody (10E8),ARP-12344 Antibody (PGT126),ARP-12474 Antibody (3BNC117),ARP-12477 Antibody (10-1074),ARP-12561 Antibody (CH01),ARP-12562 Antibody (CH02),ARP-12566 Antibody (CH106), ARP-12586 Antibody (35022), ARP-12703 Antibody (PGT145), ARP-12968 Antibody (N6). Inhouse produced monoclonal antibodies were validated by sequencing the heavy and light chain and Elisa assay. The conjugated FACS antibodies were validated either by BD (https://www.bdbiosciences.com/en-us\#) or Biolegend(https://www.biolegend.com).

\section{Eukaryotic cell lines}

Policy information about cell lines

Cell line source(s)

293T (ATCC CRL-11268)

293Freestyle (Thermo Fisher Scientific, R79007)

Expi293 (Thermo Fisher Scientific, A14528)

Homo sapiens TZM-bl Cells (ARP-8129)

Authentication

Commercial cell lines were not authenticated

Mycoplasma contamination

Mycoplasma contamination was not tested

Commonly misidentified lines (See ICLAC register)

No commonly misidentified cell lines were used

\section{Animals and other organisms}

Policy information about studies involving animals; ARRIVE guidelines recommended for reporting animal research

Laboratory animals Fourteen 6- to 10-year old outbred naive female rhesus macaques (Macaca mulatta) of Indian origin were genotyped and selected as negative for the protective major histocompatibility complex (MHC) class I alleles Mamu-A*01, Mamu-B*08, and Mamu-B*17. The animals were randomly assigned to the study groups, which were balanced for age, weight, CBC (complete blood count) and chemistry profiles. Mice were 6- to 8-week-old female BALB/c obtained from Charles River.

Wild animals No wild animals were used in the study.

Field-collected samples No field collected samples were used in the study.

Ethics oversight The macaque study protocol was approved by both the NIH and the Bioqual Institutional Animal Care and Use Committees (ACUCs). Mice experiments was approved by the Animal Care and Use Committee (ACUC) of Moderna Inc.

All animals studies were carried out in compliance with all pertinent US National Institutes of Health regulations.

Note that full information on the approval of the study protocol must also be provided in the manuscript.

\section{Flow Cytometry}

Plots

Confirm that:

\The axis labels state the marker and fluorochrome used (e.g. CD4-FITC).

\The axis scales are clearly visible. Include numbers along axes only for bottom left plot of group (a 'group' is an analysis of identical markers).

\All plots are contour plots with outliers or pseudocolor plots.

$\bigotimes$ A numerical value for number of cells or percentage (with statistics) is provided.

\section{Methodology}



R10 and stimulated with SIV Gag or HIV-1 clade B Env peptides ( $2 \mu \mathrm{g} / \mathrm{mL}$; NIH AIDS Reagent Program) in the presence of Brefeldin A (10 $\mathrm{gg} / \mathrm{mL}$; Sigma-Aldrich) and monensin (GolgiStop; BD Biosciences). Titrated amounts of anti-CD107a and antiCXCR3 were included in the culture during cell stimulation. A DMSO-only condition was included to determine background for unstimulated cells. Following stimulation for 6 hours, samples were stained with LIVE/DEAD Fixable Blue Dead Cell Stain for 10 minutes at room temperature and surface stained with titrated amounts of anti-CD14, anti-CD20, anti-CD4, antiCXCR5, anti-CD28, anti-PD-1, anti-CD8, anti-CD95 and anti-CCR7 for 20 minutes at room temperature. Cells were washed in FACS Buffer (PBS + 2\% FBS), and fixed and permeabilized (Cytofix/Cytoperm, BD Biosciences) for 20 minutes at room temperature. Cells were then washed with Perm/Wash buffer (BD Biosciences) and stained intracellularly with anti-CD3, antiCD154, anti-IFN-g, anti-IL-2, anti-IL-4, anti-IL-21 anti-TNF-a, anti-Granzyme B and anti-CD69 for 20 minutes at room temperature. Following intracellular staining, cells were washed with Perm/Wash buffer, fixed with $1 \%$ paraformaldehyde and data were acquired on a modified BD FACSymphony. Transfected adherent cells were harvested by mechanical shaking and pipetting. All the cells were washed with PBS and incubated with antibodies $(1 \mu \mathrm{g} / \mathrm{mL})$ in flow cytometry buffer $(1 \times$ PBS with $2 \% \mathrm{FBS}$, at RT for $30 \mathrm{~min}$. After washing with PBS twice, the cells were incubated with PE-conjugated goat anti-human IgG (Southern Biotech) at RT for 15 min. Adherent cells were washed once with PBS, fixed in $2 \%$ paraformaldehyde (PFA) and analyzed on a BD LSRFortessa (BD Biosciences). Data analysis and geometric mean fluorescence intensity (MFI) measurement were performed using the FlowJo software.

Instrument

Software

Cell population abundance

Gating strategy
BD LSRFortessa and BD FACSymphony (BD Biosciences).

FlowJo, version 10.7.1

All cell population tested were abundant $(>50,000$ events collected).

Appropriate gating was applied in the FSC/SSC window to exclude nonviable cell fragments and apoptotic cells. Additional gating was applied for the analysis of specific cell subpopulations.

\Tick this box to confirm that a figure exemplifying the gating strategy is provided in the Supplementary Information.

\section{Magnetic resonance imaging}

\section{Experimental design}

Design type

Design specifications

Behavioral performance measures

Acquisition

Imaging type(s)

Field strength

Sequence \& imaging parameters

Area of acquisition

Diffusion MRI

Used

Preprocessing

Preprocessing software

Normalization

Normalization template

Noise and artifact removal

Volume censoring
Indicate task or resting state; event-related or block design.

Specify the number of blocks, trials or experimental units per session and/or subject, and specify the length of each trial or block (if trials are blocked) and interval between trials.

State number and/or type of variables recorded (e.g. correct button press, response time) and what statistics were used to establish that the subjects were performing the task as expected (e.g. mean, range, and/or standard deviation across subjects). 
Statistical modeling \& inference

Model type and settings

Specify type (mass univariate, multivariate, RSA, predictive, etc.) and describe essential details of the model at the first and second levels (e.g. fixed, random or mixed effects; drift or auto-correlation).

Effect(s) tested

Define precise effect in terms of the task or stimulus conditions instead of psychological concepts and indicate whether ANOVA or factorial designs were used.

Specify type of analysis:

Whole brain $\square$ ROI-based $\square$ Both

Statistic type for inference (See Eklund et al. 2016)

Specify voxel-wise or cluster-wise and report all relevant parameters for cluster-wise methods.

Correction

Describe the type of correction and how it is obtained for multiple comparisons (e.g. FWE, FDR, permutation or Monte Carlo)

Models \& analysis

$\mathrm{n} / \mathrm{a}$ Involved in the study

$\square \square$ Functional and/or effective connectivity

$\square$ Graph analysis

$\square(\square$ Multivariate modeling or predictive analysis

Functional and/or effective connectivity

Report the measures of dependence used and the model details (e.g. Pearson correlation, partial correlation, mutual information).

Graph analysis

Report the dependent variable and connectivity measure, specifying weighted graph or binarized graph, subject-or group-level, and the global and/or node summaries used (e.g. clustering coefficient, efficiency, etc.).

Multivariate modeling and predictive analysis

Specify independent variables, features extraction and dimension reduction, model, training and evaluation metrics. 